\title{
Physical properties of dense cores in Orion B9 ${ }^{\star}$
}

\author{
O. Miettinen ${ }^{1,2}$, J. Harju ${ }^{2}$, L. K. Haikala ${ }^{2}$, and M. Juvela ${ }^{2}$ \\ ${ }^{1}$ Finnish Centre for Astronomy with ESO (FINCA), University of Turku, Väisäläntie 20, 21500 Piikkiö, Finland \\ e-mail: oskari.miettinen@helsinki.fi \\ 2 Department of Physics, PO Box 64, 00014 University of Helsinki, Finland
}

Received 3 June 2010 / Accepted 7 September 2010

\begin{abstract}
Aims. We aim to determine the physical and chemical properties of the starless and protostellar cores in Orion B9, which represents a relatively quiescent star-forming region in Orion $\mathrm{B}$.

Methods. We observed the $\mathrm{NH}_{3}(J, K)=(1,1)$ and $(2,2)$ inversion lines and the $\mathrm{N}_{2} \mathrm{H}^{+}(3-2)$ rotational lines, with the Effelsberg 100-m and APEX telescopes, respectively, towards the submillimetre peak positions in Orion B9. These data are used in conjunction with our APEX/LABOCA $870 \mu \mathrm{m}$ dust continuum data of the region.

Results. The gas kinetic temperature in the cores derived from the $\mathrm{NH}_{3}$ data is between $\sim 9.4-13.9 \mathrm{~K}$. The non-thermal velocity dispersion is subsonic in most of the cores. The non-thermal linewidth in protostellar cores appears to increase with increasing bolometric luminosity. The core masses, $\sim 2-8 M_{\odot}$, are very likely drawn from the same parent distribution as the core masses in Orion B North. Based on the virial parameter analysis, starless cores in the region are likely to be gravitationally bound, and thus prestellar. Some of the cores have a lower radial velocity than the systemic velocity of the region, suggesting that they are members of the "low-velocity part" of Orion B. The observed core-separation distances deviate from the corresponding random-like model distributions. The distances between the nearest neighbours are comparable to the thermal Jeans length. The fractional abundances of $\mathrm{NH}_{3}$ and $\mathrm{N}_{2} \mathrm{H}^{+}$in the cores are $\sim 1.5-9.8 \times 10^{-8}$ and $\sim 0.2-5.9 \times 10^{-10}$, respectively. The $\mathrm{NH}_{3}$ abundance appears to decrease with increasing $\mathrm{H}_{2}$ column and number densities. The $\mathrm{NH}_{3} / \mathrm{N}_{2} \mathrm{H}^{+}$column density ratio is larger in starless cores than in cores with embedded protostars.

Conclusions. The core population in Orion B9 is comparable in physical properties to those in nearby low-mass star-forming regions. The Orion B9 cores also seem to resemble cores found in isolation rather than those associated with clusters. Moreover, because the cores may not be randomly distributed within the region (contrary to what was suggested in our Paper I), it is unclear whether the origin of cores could be explained by turbulent fragmentation. On the other hand, many of the core properties conform to the picture of dynamic core evolution. The Orion B9 region has probably been influenced by the feedback from the nearby Ori OB $1 \mathrm{~b}$ group, and the fragmentation of the parental cloud into cores could be caused by gravitational instability.
\end{abstract}

Key words. stars: formation - ISM: clouds - ISM: individual objects: Orion B - ISM: molecules - radio lines: ISM

\section{Introduction}

The Orion B molecular cloud (L1630) is a useful target for studying dense cores and the processes of star formation. The cloud complex lies at a relatively close distance to the Sun $(\sim 450 \mathrm{pc})$, and it contains a wide range of star-forming environments, such as the high-mass star-forming region NGC 2024 and several other regions of clustered star formation (which is the dominant mode of star formation in the Galaxy), but also more quiescent areas (see, e.g., Bally 2008; Ikeda et al. 2009; Buckle et al. 2010). We recently mapped region of the central part of Orion B of about $36^{\prime} \times 27^{\prime}(4.7 \mathrm{pc} \times 3.5 \mathrm{pc})$, called Orion B9 $\left(l=206^{\circ} .1, b=-15^{\circ} .8\right)$, at $870 \mu \mathrm{m}$ dust continuum using LABOCA on APEX, and discovered 12 dense submm cores (Miettinen et al. 2009; hereafter Paper I). The cores were classified into starless and protostellar ones by using the Spitzer data, yielding the result that half the cores have embedded

* This publication is based on data acquired with the Atacama Pathfinder Experiment (APEX) under programme 084.F-9312, and observations with the 100-m telescope of the Max-Planck-Institut für Radioastronomie (MPIfR) at Effelsberg. APEX is a collaboration between the MPIfR, the European Southern Observatory, and the Onsala Space Observatory. protostar(s). In the present paper, we examine the physical characteristics of the Orion B9 cores further. To determine the gas kinetic temperature, kinematics, and the dynamical state of the cores, we performed $\mathrm{NH}_{3}(1,1)$ and $(2,2)$, and $\mathrm{N}_{2} \mathrm{H}^{+}(3-2)$ observations towards the core submm peak positions with the Effelsberg 100-m and APEX telescopes, respectively. In this paper, the derived temperatures are also used to recalculate the temperature-dependent core parameters presented in Paper I.

This paper is organised as follows. The observations and data-reduction procedures are described in Sect. 2. The observational results are presented in Sect. 3. In Sect. 4, we describe the analysis of the physical and chemical properties of the cores. Discussion of the results is given in Sect. 5, and in Sect. 6, we summarise our main conclusions.

\section{Observations and data reduction}

\subsection{Effelsberg 100-m observations}

Pointed observations of the $\mathrm{NH}_{3}(1,1)$ and $(2,2)$ inversion line emission towards the dense cores in Orion B9 were performed with the Effelsberg 100-m telescope of the MPIfR on 2009 November 23-25. The target positions listed in Table 1 were 
Table 1. Submillimetre cores in Orion B9.

\begin{tabular}{|c|c|c|c|}
\hline \multirow{2}{*}{$\begin{array}{c}\text { Source } \\
\text { name }\end{array}$} & \multicolumn{2}{|c|}{ Peak position } & \multirow[t]{2}{*}{ Class } \\
\hline & $\alpha_{2000.0}[\mathrm{~h}: \mathrm{m}: \mathrm{s}]$ & $\delta_{2000.0}\left[{ }^{\circ}:^{\prime \prime}:^{\prime \prime}\right]$ & \\
\hline IRAS 05399-0121 & 054227.4 & -011950 & $0 / \mathrm{I}$ \\
\hline SMM 1 & 054230.5 & -012045 & prestella1 \\
\hline SMM 2 & 054232.9 & -012528 & prestellar \\
\hline SMM 3 & 054244.4 & -011603 & 0 \\
\hline IRAS 05405-0117 & 054302.7 & -011621 & 0 \\
\hline SMM 4 & 054303.9 & -011544 & 0 \\
\hline SMM 5 & 054304.5 & -011706 & prestellar \\
\hline SMM 6 & 054305.1 & -011838 & prestellar \\
\hline Ori B9 N & 054305.7 & -011441 & prestellar \\
\hline SMM 7 & 054322.1 & -011346 & prestellar \\
\hline IRAS 05412-0105 & 054346.4 & -010430 & 0 \\
\hline IRAS 05413-0104 & 054351.3 & -010250 & 0 \\
\hline
\end{tabular}

drawn from the submm peak positions in the LABOCA $870 \mu \mathrm{m}$ dust continuum map of the region. In Table 1, we also show the source classification from Paper I.

The $1.3 \mathrm{~cm}$ HEMT receiver was tuned to a frequency of $23708.564 \mathrm{MHz}$, lying midway between the rest frequencies of the $\mathrm{NH}_{3}(1,1)$ and $(2,2)$ lines, which are separated by about $28 \mathrm{MHz}$. For the sky frequency conversion the local standard of rest (LSR) velocity was set to $9.2 \mathrm{~km} \mathrm{~s}^{-1}$. The receiver measures orthogonal linear polarisations. The sum of the two channels was fed into the Fast Fourier Transfrom Spectrometer (FFTS) with a $100 \mathrm{MHz}$ bandwidth. In this manner, the Stokes $I$ spectra of the two lines were measured simultaneously. The backend is a modified copy of the FFTS at the APEX telescope (see Sect. 2.2). The $100 \mathrm{MHz}$ band was split into 16384 channels. This resulted in a channel separation of $6.1 \mathrm{kHz}$ which corresponds to about $77 \mathrm{~m} \mathrm{~s}^{-1}$ at $23.7 \mathrm{GHz}$. The spectral resolution (equivalent noise bandwidth) is about $9.8 \mathrm{kHz}\left(124 \mathrm{~m} \mathrm{~s}^{-1}\right)$. One of the sources, SMM 6, was also observed in $\mathrm{NH}_{3}(1,1)(23694.4955 \mathrm{MHz})$ using a $20 \mathrm{MHz}$ bandwidth to resolve the hyperfine structure in more detail. In this configuration, the channel separation is $1.22 \mathrm{kHz}\left(15 \mathrm{~m} \mathrm{~s}^{-1}\right)$.

The FWHM (full width at half maximum) beamsize at the observed frequencies is $40^{\prime \prime}(0.09 \mathrm{pc}$ at $450 \mathrm{pc})$, and the main beam efficiency is $\eta_{\mathrm{MB}}=0.52$. Observations were conducted in frequency switching mode with a frequency throw of $6 \mathrm{MHz}$. The single-sideband (SSB) system temperatures were about $200-310 \mathrm{~K}$ in the main-beam brightness temperature, $T_{\mathrm{MB}}$, scale during the observations. Typical integration time was $\sim 120 \mathrm{~min}$ per position, resulting in a $1 \sigma \mathrm{rms}$ noise of about $38-75 \mathrm{mK}$. For further information of the telescope and the receiving system, see http : //www . mpifr-bonn .mpg.de/radioteleskop/.

The $\mathrm{NH}_{3}(1,1)$ and $(2,2)$ emission was detected towards all submm cores except IRAS 05412-0105 (rms $79 \mathrm{mK}$ ). This source was also very weak $\left(0.17 \mathrm{Jy} \mathrm{beam}^{-1}\right)$ in our LABOCA $870 \mu \mathrm{m}$ map (Paper I; Fig. 1 therein). We performed test measurements of the $\mathrm{NH}_{3}(3,3)$ inversion line at $23870.1292 \mathrm{MHz}$ towards two strong $\mathrm{NH}_{3}(1,1)$ sources in our sample: SMM 4 and IRAS 05405-0117. No lines were detected after $30 \mathrm{~min}$ integration per position ( $\mathrm{rms} \sim 200-300 \mathrm{mK}$ at the velocity resolution $15 \mathrm{~m} \mathrm{~s}^{-1}$ ).

Telescope pointing and focus were checked about every $1-1.5 \mathrm{~h}$ by continuum scans on radio quasars PKS 0420-014 and $3 \mathrm{C} 147$, and the radio galaxy $3 \mathrm{C} 213$. The pointing was typically accurate to within $\sim 7^{\prime \prime}$. Absolute flux calibration was based upon continuum cross scans on the planetary nebula NGC 7027, and the radio galaxies/quasars 3C 123, 3C 147, and 3C 286, for which we adopted the flux densities $5.58 \pm 0.10$,
$2.93,1.91 \pm 0.11$, and $2.46 \pm 0.05 \mathrm{Jy}$ at $1.3 \mathrm{~cm}$, respectively (Ott et al. 1994; Peng et al. 2000). We were not able to retrieve the zenith opacity, $\tau_{\mathrm{z}}$, by doing a sky-dipping measurement or by observing calibrators in good weather in a wide range of elevations (e.g., Pillai et al. 2006; Frieswijk et al. 2007). Instead, we estimated the average $\tau_{\mathrm{z}}$ at $1.3 \mathrm{~cm}$ by fitting all the calibration measurements with the function $G(\theta) \times \mathrm{e}^{-\tau_{z} / \sin \theta}$, where $G(\theta)$ is the instrumental gain-elevation curve given on the telescope www pages (see above). In this manner, we obtained an average $\tau_{\mathrm{z}}$ of 0.15 . This value is consistent with previous estimates at centrimetre wavelengths (Frieswijk et al. 2007; Appendix A therein). The typical uncertainty in absolute flux calibration is $\sim 15 \%$ (excluding the systematic error in $\tau_{\mathrm{z}}$ ). Note that this uncertainty does not affect the parameters that depend on the line intensity ratios (e.g, optical thickness, kinetic temperature), or the kinematical parameters (centroid velocity and width of the line). The calibration uncertainty propagates to the values of the line excitation temperature, and $\mathrm{NH}_{3}$ column densities and fractional abundances (see Sect. 4.1).

The spectra were reduced using the CLASS package of the IRAM's GILDAS software ${ }^{1}$. For a given source, the spectra were averaged and folded. A first order polynomial, and in one case (SMM 4) a third order polynomial, was applied to correct the baseline in the $\mathrm{NH}_{3}(1,1)$ lines. A polynomial baseline of order 3 was subtracted from the $\mathrm{NH}_{3}(2,2)$ lines. We fitted the hyperfine structure of the $\mathrm{NH}_{3}(1,1)$ line using "method $\mathrm{NH} 3(1,1)$ " of the CLASS package to derive the LSR velocity $\left(v_{\mathrm{LSR}}\right)$ of the emission, $F W H M$ linewidth $(\Delta v)$, and the line optical thickness (see Sect. 3.1). The emission of the $(2,2)$ satellite lines was not detected. Nevertheless, the radial velocities and linewidths were determined using "method NH3 $(2,2)$ " of CLASS.

\subsection{APEX observation}

The $\mathrm{N}_{2} \mathrm{H}^{+}(3-2)$ observations towards nine cores in the central region of Orion B9 (i.e., IRAS 05399-0121 and 054050117, SMM 1, and 3-7, and Ori B9 N) were carried out on 2009 September 2 and 11 with the APEX telescope ${ }^{2}$. The backend was the MPIfR FFTS (Klein et al. 2006) with a $1 \mathrm{GHz}$ bandwidth divided into 8192 channels. The resulting channel width was $122 \mathrm{kHz}$ which corresponds to $0.13 \mathrm{~km} \mathrm{~s}^{-1}$ at the observed frequency $279.5 \mathrm{GHz}$. As frontend, we used APEX-2 of the Swedish Heterodyne Facility Instrument (SHFI; Vassilev et al. 2008). At $279.5 \mathrm{GHz}$, the APEX beamsize is $\simeq 22^{\prime \prime}$. 3 , and the main beam efficiency is $\eta_{\mathrm{MB}} \simeq 0.74$.

The observations were performed in the wobbler-switching mode with a $100^{\prime \prime}$ throw (symmetric offsets) and a chopping rate of $0.5 \mathrm{~Hz}$. The typical total integration time was $\sim 11 \mathrm{~min}$ per position, and the SSB system temperature was between $210-360 \mathrm{~K}$ ( $T_{\mathrm{MB}}$ scale) during these observations. The telescope pointing was checked using the planets Mars and Uranus, and the stars $\alpha$ Orionis and V1259 Ori, and was found to be accurate to $\sim 3^{\prime \prime}$. The calibration was achieved by the chopper-wheel method, and the intensity scale given by the system is $T_{\mathrm{A}}^{*}$, the antenna temperature corrected for atmospheric attenuation. The observed intensities were converted to the main-beam brightness temperature scale by $T_{\mathrm{MB}}=T_{\mathrm{A}}^{*} / \eta_{\mathrm{MB}}$.

The spectra were reduced using the CLASS. The individual spectra were averaged, and linear baselines were subtracted from

\footnotetext{
1 http://wWW . iram. fr/IRAMFR/GILDAS

2 Other spectral lines observed under this programme include $\mathrm{C}^{17} \mathrm{O}(2-1), \mathrm{N}_{2} \mathrm{D}^{+}(3-2), \mathrm{DCO}^{+}(4-3)$, and $\mathrm{H}^{13} \mathrm{CO}^{+}(4-3)$. These observations will be presented in the forthcoming paper.
} 
the resulting sum spectra. The resulting $1 \sigma \mathrm{rms}$ noise values are $35-130 \mathrm{mK}$.

The $J=3-2$ transition of $\mathrm{N}_{2} \mathrm{H}^{+}$contains 38 hyperfine components. We fitted the hyperfine structure of the lines using "method hfs" of the CLASS. For the rest frequencies of the hyperfine components, we used the values from Pagani et al. (2009, Table 4 therein). The adopted central frequency, $279511.832 \mathrm{MHz}$, is that of the $J_{F_{1} F}=3_{45} \rightarrow 2_{34}$ hyperfine component which has a relative intensity of $17.46 \%$

\section{Observational results}

\section{1. $\mathrm{NH}_{3}$}

The $\mathrm{NH}_{3}(1,1)$ and $(2,2)$ spectra are shown in Fig. 1. The $\mathrm{NH}_{3}(1,1)$ spectrum observed from SMM 6 with a $20 \mathrm{MHz}$ bandwidth (see Sect. 2.1) is shown in Fig. 2. In Table 2, we give the line parameters resulting from the hyperfine/Gaussian fits to the $\mathrm{NH}_{3}$ lines. The so-called "main group optical thickness", $\tau_{\mathrm{m}}$, given in Table 2 is the sum of peak optical thicknesses of the eight hyperfine components included in the main group. For the $(1,1)$ transition this equals to the corresponding sum of the ten satellite components, so the total of all peak optical thicknesses is $2 \times \tau_{\mathrm{m}}$. Assuming that the line profile (due to velocity dispersion) is Gaussian, the integrated optical thickness can be obtained from $\int \tau(v) \mathrm{d} v=\sqrt{\pi / \ln 2} \tau_{\mathrm{m}} \Delta v$, where $\Delta v$ is the linewidth $(F W H M)$ in velocity units. The uncertainties associated with $v_{\mathrm{LSR}}, \Delta v$, and $\tau_{\mathrm{m}}$ are the $1 \sigma$ fitting errors. The uncertainty in $T_{\mathrm{MB}}, \sigma\left(T_{\mathrm{MB}}\right)$, includes the absolute calibration uncertainty of $15 \%$, and the $1 \sigma \mathrm{rms}$ noise in the spectrum, and was obtained by quadratic summing of these two errors as $\sigma\left(T_{\mathrm{MB}}\right)=\sqrt{\sigma_{\text {cal }}^{2}+\sigma_{\text {rms }}^{2}}$. The determination of the line excitation temperature, $T_{\mathrm{ex}}$, listed in the last column of Table 2, is described in Sect. 4.1.1.

The $\mathrm{NH}_{3}$ spectra towards SMM 4 and Ori B9 N show evidence of a second velocity component in addition to the strong line at about $9.1 \mathrm{~km} \mathrm{~s}^{-1}$ corresponding to the systemic velocity of Orion B. In the case of IRAS 05405-0117, there is also a hint of a second velocity component, probably resulting from the fact that the $40^{\prime \prime}$ beam slightly overlaps with the nearby core SMM 4. In the direction of SMM 4, the second component has a velocity of about $1.6 \mathrm{~km} \mathrm{~s}^{-1}$, whereas towards Ori $\mathrm{B} 9 \mathrm{~N}$ the velocity is $1.9 \mathrm{~km} \mathrm{~s}^{-1}$. In both cases, the main hyperfine group of the second velocity component overlaps with the inner satellite line $F_{1}=1-2$ of the principal velocity component. Consequently, the inner satellite line $F_{1}=2-1$ of the second velocity component contaminates the main hyperfine group of the principal velocity component. These spectra were analysed using a two-component fit to the $\mathrm{NH}_{3}(1,1)$ hyperfine structure. We note that the secondary velocity components towards SMM 4 and Ori B9 $\mathrm{N}$ are close to the radial velocities of IRAS 05413$0104\left(1.5 \mathrm{~km} \mathrm{~s}^{-1}\right)$ and SMM $7\left(3.6 \mathrm{~km} \mathrm{~s}^{-1}\right)$. We also note that the second velocity components influence the core parameters derived from the dust continuum emission (see Sect. 4.3). Part of the observed flux density may be due to the dust component associated with another source along the line of sight. However, because continuum observations do not provide velocity information, it is impossible to solve this "overlap" problem.

The $\mathrm{NH}_{3}(1,1)$ line towards SMM 6 measured using a $20 \mathrm{MHz}$ bandwidth is $30 \mathrm{~m} \mathrm{~s}^{-1}$ narrower and has a $15 \%$ larger optical thickness than that measured using a $100 \mathrm{MHz}$ bandwidth. The difference in the linewidths corresponds to effect expected from instrumental broadening with the two configurations. As is expected, the product $\Delta v \tau_{\mathrm{m}}$ is roughly constant at the two spectral resolutions (see Eq. (5)). As the high resolution spectrum is only available for one object, we use in the subsequent analysis the $100 \mathrm{MHz}$ spectra for all of them.

\section{2. $\mathrm{N}_{2} \mathrm{H}^{+}$}

The $\mathrm{N}_{2} \mathrm{H}^{+}(3-2)$ spectra are shown in Fig. 3. In Table 3, we show the line parameters obtained from the fits to the hyperfine structure. Here, the value of $\sigma\left(T_{\mathrm{MB}}\right)$ includes only the $1 \sigma$ rms noise in the spectrum (Col. (4) of Table 3 ).

The LSR velocities of the $\mathrm{N}_{2} \mathrm{H}^{+}(3-2)$ lines are mostly similar to those determined from $\mathrm{NH}_{3}(1,1)$, as can be seen in the top panel of Fig. 4. On the other hand, there is hardly any correlation between the $\mathrm{N}_{2} \mathrm{H}^{+}(3-2)$ and $\mathrm{NH}_{3}(1,1)$ linewidths (bottom panel of Fig. 4). It should be noted, however, that the hyperfine fitting to the $\mathrm{N}_{2} \mathrm{H}^{+}$spectra is uncertain due to strongly overlapping components and rather a poor signal-to-noise $(\mathrm{S} / \mathrm{N})$ ratio.

The $\mathrm{N}_{2} \mathrm{H}^{+}(3-2)$ spectra towards SMM 4 and Ori B9 $\mathrm{N}$ show the same lower radial velocity components $\left(\$ 2 \mathrm{~km} \mathrm{~s}^{-1}\right)$ as seen in $\mathrm{NH}_{3}(1,1)$. On the other hand, the "principal component" at $\sim 9 \mathrm{~km} \mathrm{~s}^{-1}$ is not detected towards SMM 4 and it is also very weak towards Ori B9 N. Similarly, in Paper I we found that the $\mathrm{N}_{2} \mathrm{H}^{+}(1-0)$ spectra towards the selected position near IRAS 05405-0117/SMM 4 and Ori B9 N have second velocity components at $\sim 1.3$ and $\sim 2.2 \mathrm{~km} \mathrm{~s}^{-1}$, respectively. The latter position also had additional $\mathrm{N}_{2} \mathrm{D}^{+}(2-1)$ line centred at $\sim 2.3 \mathrm{~km} \mathrm{~s}^{-1}$.

We note that Harju et al. (2006) probably detected $\mathrm{N}_{2} \mathrm{H}^{+}$(4-3) towards IRAS 05405-0117. They were using a double sideband system where a coincidence with $\mathrm{DCO}^{+}(5-4)$ from image band could not be ruled out. Using the rest frequencies of $\mathrm{N}_{2} \mathrm{H}^{+}$rotational lines from Pagani et al. (2009), the peak velocity of the suggested $\mathrm{N}_{2} \mathrm{H}^{+}(4-3)$ line $\left(9.25 \mathrm{~km} \mathrm{~s}^{-1}\right)$ is very similar to that of $\mathrm{N}_{2} \mathrm{H}^{+}(3-2)\left(9.30 \mathrm{~km} \mathrm{~s}^{-1}\right)$. For $\mathrm{DCO}^{+}(5-4)$ the peak velocity would be about $0.3 \mathrm{~km} \mathrm{~s}^{-1}$ lower $\left(8.95 \mathrm{~km} \mathrm{~s}^{-1}\right)$. The $\mathrm{N}_{2} \mathrm{H}^{+}(4-3)$ linewidth $\left(0.34 \mathrm{~km} \mathrm{~s}^{-1}\right)$ is similar to that of $\mathrm{NH}_{3}(1,1)\left(0.32 \mathrm{~km} \mathrm{~s}^{-1}\right)$.

\section{Analysis}

\section{1. $\mathrm{NH}_{3}$ analysis}

In this subsection, we derive physical parameters from $\mathrm{NH}_{3}$ data. The obtained results are given in Col. (7) of Table $2\left(T_{\mathrm{ex}}\right)$, and in Table 4. The analysis follows the concept first presented in Ho et al. (1979) and further discussed in Ho et al. (1983). Here it is assumed that the same excitation temperature, $T_{\mathrm{ex}}$, characterises all the hyperfine transitions between the $(1,1)$ and $(2,2)$ inversion doublets.

\subsubsection{Excitation, rotation, and kinetic temperatures}

The $T_{\text {ex }}$ was calculated at the $(1,1)$ line peak using the optical thickness, $\tau_{\text {peak }}$, at that velocity, from the equation

$T_{\mathrm{ex}}=\frac{T_{11}}{\ln \left[1+\frac{T_{\mathrm{MB}}(v)}{T_{11}} \frac{1}{1-\mathrm{e}^{-\tau(v)}}+F\left(T_{\mathrm{bg}}\right)\right]}$,

where $T_{11}=h v_{11} / k_{\mathrm{B}}, h$ is the Planck constant, $v_{11}$ is the $(1,1)$ line frequency, $k_{\mathrm{B}}$ is the Boltzmann constant, $T_{\mathrm{bg}}=2.73 \mathrm{~K}$ is the background temperature, and $F(T)=\left(\mathrm{e}^{h v / k_{\mathrm{B}} T}-1\right)^{-1}$. The uncertainty in $T_{\mathrm{ex}}$ was calculated by propagating the errors in 

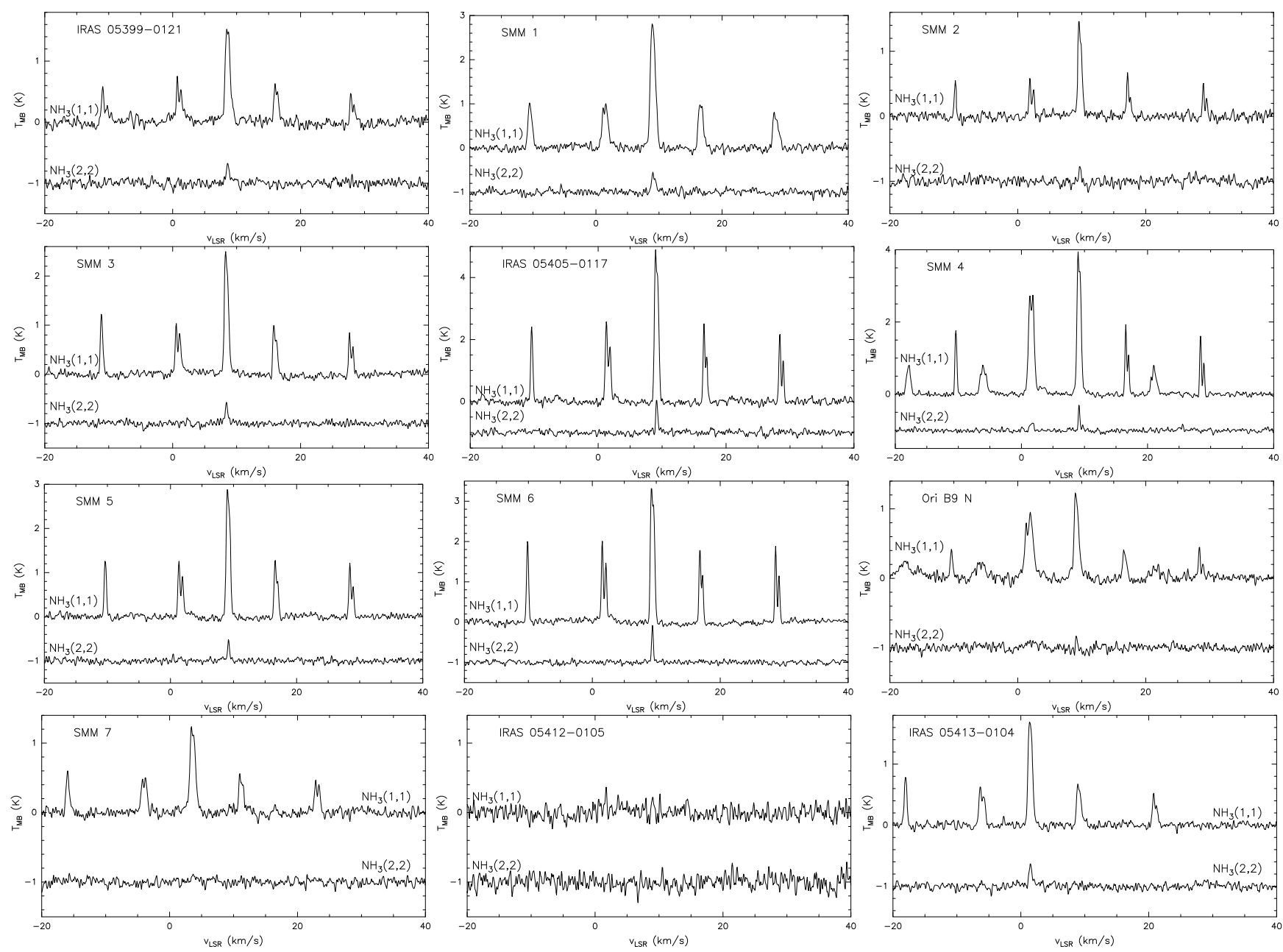

Fig. 1. $\mathrm{NH}_{3}(1,1)$ and $(2,2)$ spectra measured from the pre- and protostellar cores in Orion B9. The temperature scale is in $T_{\mathrm{MB}} \cdot \mathrm{NH}_{3}(2,2)$ spectra are offset by $-1 \mathrm{~K}$ from zero baseline for clarity. Note that there are two velocity components towards SMM 4 and Ori B9 N, and that no lines were detected towards IRAS 05412-0105.

the peak values of $T_{\mathrm{MB}}(v)$ and $\tau(v)$. The $\tau(v)$ distribution was obtained using the $(1,1)$ hyperfine fit from CLASS which yields the LSR velocity line centroid, the width of an individual hyperfine component, and the main group optical thickness, $\tau_{\mathrm{m}}$ (see Sect. 3.1).

The rotational temperature, $T_{\text {rot }}$, can be in principle calculated using the formula

$T_{\text {rot }}=\frac{-41.5}{\ln \left[\frac{3}{5} \frac{N(2,2)}{N(1,1)}\right]}$,

where $N(1,1)$ and $N(2,2)$ are the column densities of ammonia molecules at the $(1,1)$ and $(2,2)$ rotational levels, respectively. The estimation of these column densities is described in Sect. 4.1.2. The ratio $3 / 5$ refers to the ratio of the statistical weights of the two states $(g(1,1) / g(2,2))$.

When the optical thickness, $\tau_{\mathrm{m}}$, is known, Eq. (2) can be written as

$T_{\text {rot }}=\frac{-41.5}{\ln \left\{\frac{-0.283}{\tau_{\mathrm{m}}} \ln \left[1-\frac{T_{\mathrm{MB}}(2,2)}{T_{\mathrm{MB}}(1,1)}\left(1-\mathrm{e}^{-\tau_{\mathrm{m}}}\right)\right]\right\}}$

(Ho et al. 1983; their Eq. (4)). Here it is assumed that $T_{\mathrm{ex}}$ and $\Delta v$ are the same for both the $(1,1)$ and $(2,2)$ transitions. The error associated with $T_{\text {rot }}$ was propagated from the uncertainties in $T_{\mathrm{MB}}(1,1), T_{\mathrm{MB}}(2,2)$, and $\tau_{\mathrm{m}}$. When $\tau_{\mathrm{m}}$ could not be reliably determined (SMM 4, Ori B9 N, and their additional velocity components), we estimated the $\mathrm{NH}_{3}(1,1)$ and $\mathrm{NH}_{3}(2,2)$ column densities from the integrated intensities assuming $T_{\mathrm{ex}}=5 \mathrm{~K}$, as described below. In these cases, $T_{\text {rot }}$ was also calculated using Eq. (2), and the uncertainty in $T_{\text {rot }}$ was propagated from the uncertainties in the column densities. The values of $T_{\text {rot }}$ calculated using Eq. (2) are mostly similar to those resulting from Eq. (3) within the errors (see Table 4). The subsequent analysis includes only those $T_{\text {rot }}$ values which could be derived using Eq. (3).

The gas kinetic temperature, $T_{\text {kin }}$, was calculated from $T_{\text {rot }}$ using the relationship given by Tafalla et al. (2004):

$T_{\text {kin }}=\frac{T_{\text {rot }}}{1-\frac{T_{\text {rot }}}{42} \ln \left(1+1.1 \mathrm{e}^{-\frac{16}{T_{\text {rot }}}}\right)}$.

This relationship is recommended for dense cores with gas temperatures $\$ 20 \mathrm{~K}$. Uncertainty in $T_{\text {kin }}$ was propagated from the uncertainty in $T_{\text {rot }}$.

\subsection{2. $\mathrm{NH}_{3}$ column density calculations}

The column density in the $(J, K)=(1,1)$ state was calculated using the formula (see Harju et al. 1993; Eqs. (3) and (5) therein)

$$
\begin{gathered}
N(1,1)=\frac{3 h \epsilon_{0}}{2 \pi^{2} \mu^{2}} \frac{\sqrt{\pi}}{2 \sqrt{\ln 2}} \frac{J(J+1)}{K^{2}} F\left(T_{\text {ex }}\right)\left(\mathrm{e}^{T_{11} / T_{\text {ex }}}+1\right) \Delta v \tau_{\text {tot }} \\
=7.83 \times 10^{12} F\left(T_{\text {ex }}\right)\left(\mathrm{e}^{1.14 / T_{\text {ex }}}+1\right) \Delta v\left[\mathrm{~km} \mathrm{~s}^{-1}\right] \tau_{\text {tot }} \mathrm{cm}^{-2},
\end{gathered}
$$


Table 2. $\mathrm{NH}_{3}(1,1)$ and $(2,2)$ line parameters.

\begin{tabular}{|c|c|c|c|c|c|c|}
\hline Source & $\begin{array}{l}\text { Transition } \\
(J, K)\end{array}$ & $\begin{array}{c}v_{\mathrm{LSR}} \\
{\left[\mathrm{km} \mathrm{s}^{-1}\right]}\end{array}$ & $\begin{array}{c}\Delta v \\
{\left[\mathrm{~km} \mathrm{~s}^{-1}\right]}\end{array}$ & $\begin{array}{c}T_{\mathrm{MB}}{ }^{a} \\
{[\mathrm{~K}]}\end{array}$ & $\tau_{\mathrm{m}}{ }^{b}$ & $\begin{array}{l}T_{\mathrm{ex}} \\
{[\mathrm{K}]}\end{array}$ \\
\hline \multirow[t]{2}{*}{ IRAS 05399-0121 } & $(1,1)$ & $8.61 \pm 0.01$ & $0.59 \pm 0.02$ & $1.6 \pm 0.2$ & $1.10 \pm 0.13$ & $5.4 \pm 0.5$ \\
\hline & $(2,2)$ & $8.64 \pm 0.02$ & $0.42 \pm 0.08$ & $0.3 \pm 0.1$ & & \\
\hline \multirow[t]{2}{*}{ SMM 1} & $(1,1)$ & $9.04 \pm 0.003$ & $0.67 \pm 0.01$ & $2.9 \pm 0.4$ & $1.23 \pm 0.06$ & $7.2 \pm 0.7$ \\
\hline & $(2,2)$ & $9.13 \pm 0.02$ & $0.53 \pm 0.05$ & $0.4 \pm 0.1$ & & \\
\hline \multirow[t]{2}{*}{ SMM 2} & $(1,1)$ & $9.71 \pm 0.003$ & $0.34 \pm 0.01$ & $1.4 \pm 0.2$ & $1.38 \pm 0.16$ & $5.3 \pm 0.4$ \\
\hline & $(2,2)$ & $9.72 \pm 0.02$ & $0.26 \pm 0.04$ & $0.2 \pm 0.1$ & & \\
\hline \multirow[t]{2}{*}{ SMM 3} & $(1,1)$ & $8.40 \pm 0.002$ & $0.38 \pm 0.01$ & $2.5 \pm 0.4$ & $2.14 \pm 0.10$ & $6.1 \pm 0.5$ \\
\hline & $(2,2)$ & $8.42 \pm 0.01$ & $0.42 \pm 0.06$ & $0.4 \pm 0.1$ & & \\
\hline \multirow[t]{2}{*}{ IRAS 05405-0117 } & $(1,1)$ & $9.12 \pm 0.001$ & $0.32 \pm 0.003$ & $4.9 \pm 0.7$ & $3.36 \pm 0.09$ & $8.4 \pm 0.9$ \\
\hline & $(2,2)$ & $9.17 \pm 0.01$ & $0.30 \pm 0.02$ & $1.1 \pm 0.2$ & & \\
\hline \multirow[t]{2}{*}{ SMM 4} & $(1,1)$ & $9.12 \pm 0.001$ & $0.34 \pm 0.01$ & $3.2 \pm 0.5^{c}$ & $1.33 \pm 0.03$ & $8.6 \pm 0.9$ \\
\hline & $(2,2)$ & $9.15 \pm 0.01$ & $0.23 \pm 0.02$ & $0.7 \pm 0.1$ & & \\
\hline \multirow[t]{2}{*}{ SMM 4 (2nd v-comp.) } & $(1,1)$ & $1.62 \pm 0.003$ & $0.71 \pm 0.01$ & $2.0 \pm 0.2$ & $1.50 \pm 0.06$ & $5.1 \pm 0.5$ \\
\hline & $(2,2)$ & $1.66 \pm 0.03$ & $0.51 \pm 0.07$ & $0.2 \pm 0.1$ & & \\
\hline \multirow[t]{2}{*}{ SMM 5} & $(1,1)$ & $9.16 \pm 0.002$ & $0.35 \pm 0.004$ & $3.0 \pm 0.5$ & $2.28 \pm 0.08$ & $6.6 \pm 0.6$ \\
\hline & $(2,2)$ & $9.21 \pm 0.01$ & $0.31 \pm 0.03$ & $0.5 \pm 0.1$ & & \\
\hline \multirow[t]{2}{*}{ SMM 6} & $(1,1)$ & $9.38 \pm 0.001$ & $0.29 \pm 0.002$ & $3.4 \pm 0.5$ & $4.66 \pm 0.11$ & $6.3 \pm 0.5$ \\
\hline & $(2,2)$ & $9.41 \pm 0.004$ & $0.30 \pm 0.01$ & $0.9 \pm 0.1$ & & \\
\hline SMM 6 (20 MHz BW) & $(1,1)$ & $9.44 \pm 0.001$ & $0.26 \pm 0.002$ & $3.3 \pm 0.5$ & $5.38 \pm 0.13$ & $6.2 \pm 0.5$ \\
\hline \multirow[t]{2}{*}{ Ori B9 N } & $(1,1)$ & $9.14 \pm 0.004$ & $0.40 \pm 0.01$ & $1.1 \pm 0.2^{c}$ & $1.08 \pm 0.15$ & $5.0 \pm 0.4$ \\
\hline & $(2,2)$ & $9.18 \pm 0.02$ & $0.21 \pm 0.04$ & $0.2 \pm 0.05$ & & \\
\hline \multirow[t]{2}{*}{ Ori B9 N (2nd v-comp.) } & $(1,1)$ & $1.93 \pm 0.01$ & $1.50 \pm 0.04$ & $0.7 \pm 0.1$ & $0.37 \pm 0.09$ & $5.1 \pm 0.6$ \\
\hline & $(2,2)$ & $2.29 \pm 0.11$ & $2.53 \pm 0.28$ & $0.1 \pm 0.04$ & & \\
\hline \multirow[t]{2}{*}{ SMM 7} & $(1,1)$ & $3.60 \pm 0.01$ & $0.60 \pm 0.02$ & $1.2 \pm 0.2$ & $2.14 \pm 0.14$ & $4.1 \pm 0.2$ \\
\hline & $(2,2)$ & $3.89 \pm 0.10$ & $0.67 \pm 0.14$ & $0.1 \pm 0.05$ & & \\
\hline \multirow{2}{*}{ IRAS 05413-0104 } & $(1,1)$ & $1.48 \pm 0.003$ & $0.46 \pm 0.01$ & $1.8 \pm 0.3$ & $1.68 \pm 0.09$ & $5.2 \pm 0.4$ \\
\hline & $(2,2)$ & $1.52 \pm 0.02$ & $0.53 \pm 0.04$ & $0.4 \pm 0.1$ & & \\
\hline
\end{tabular}

Notes. ${ }^{(a)}$ The error in $T_{\mathrm{MB}}$ includes the $\sim 15 \%$ calibration uncertainty (see the text in Sect. 3.1$) .{ }^{(b)} \tau_{\mathrm{m}}$ is the optical thickness of the (1, 1) main group. The total optical thickness, i.e., the sum over all satellites, is $\tau_{\text {tot }}=2 \times \tau_{\mathrm{m}} \cdot{ }^{(c)} T_{\mathrm{MB}}(1,1)$ was determined by substracting the second velocity component from the spectrum.

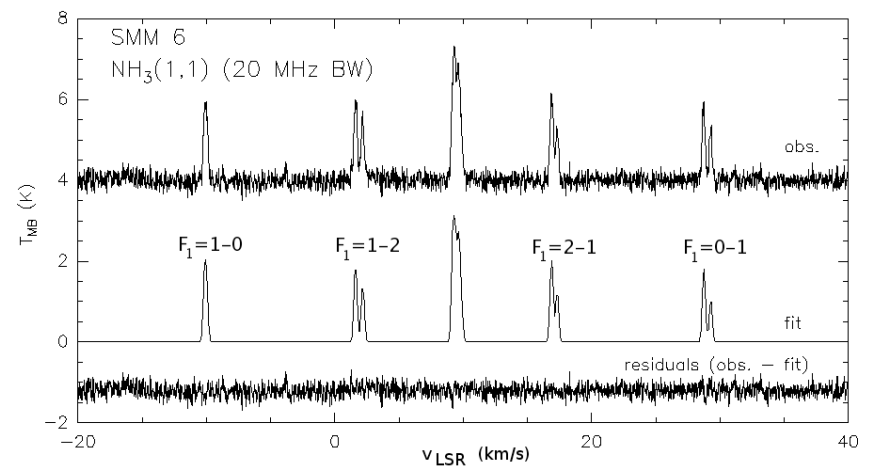

Fig. 2. $\mathrm{NH}_{3}(1,1)$ spectrum measured from SMM 6 using the FFTS with a $20 \mathrm{MHz}$ bandwidth. Below the spectrum are shown the model fit to the hyperfine structure and the residual spectrum. Satellite hyperfine components in the $F_{1}^{\prime} \rightarrow F_{1}$ format are indicated.

where $\epsilon_{0}$ is the vacuum permittivity, $\mu$ is the permanent electric dipole moment (1.476 D), and $\tau_{\text {tot }}=2 \times \tau_{\mathrm{m}}$.

For SMM 4 and Ori B9 N, and their second velocity components (for which $\tau_{\mathrm{m}}$ is uncertain in all the cases), $N(1,1)$ was also calculated by assuming optically thin emission $(\tau \ll 1)$, and using the integrated intensities of clean satellites, i.e., those where the two velocity components do not overlap. There are three satellite lines in the $\mathrm{NH}_{3}(1,1)$ spectra of both SMM 4 and Ori B9 $\mathrm{N}$ that do not suffer from the contamination of the second velocity component, i.e., $F_{1}=1-0,2-1$, and $0-1$ (see Fig. 1 ). For the second velocity components, the corresponding satellite lines are $F_{1}=1-0,1-2$, and $0-1$. Taking into account that these lines comprise $R_{i}=36.1 \%$ of the total line strength, and assuming that the filling fraction of the emission in the beam is $\eta_{\mathrm{f}}=1$, we can derive the formula

$$
\begin{gathered}
N(1,1)=\frac{3 k_{\mathrm{B}} \epsilon_{0}}{2 \pi^{2} \mu^{2} v_{11}} \frac{J(J+1)}{K^{2}} \frac{\mathrm{e}^{T_{11} / T_{\mathrm{ex}}}+1}{1-\frac{F\left(T_{\mathrm{bg}}\right)}{F\left(T_{\mathrm{ex}}\right)}} \frac{1}{R_{i}} \int T_{\mathrm{MB}}(1,1 ; \mathrm{s}) \mathrm{d} v \\
=6.47 \times 10^{12} \frac{\mathrm{e}^{1.14 / T_{\mathrm{ex}}}+1}{1-\frac{F\left(T_{\mathrm{bg}}\right.}{F\left(T_{\mathrm{ex}}\right)}} \frac{1}{R_{i}} \int T_{\mathrm{MB}}(1,1 ; \mathrm{s}) \mathrm{d} v\left[\mathrm{~K} \mathrm{~km} \mathrm{~s}^{-1}\right] \mathrm{cm}^{-2},
\end{gathered}
$$

where $T_{\mathrm{MB}}$ is integrated over the three above mentioned satellite lines (the integrated satellite intensities are $2.53 \pm 0.03$ and $0.74 \pm 0.03 \mathrm{~K} \mathrm{~km} \mathrm{~s}^{-1}$ for SMM 4 and Ori B9 N, respectively; for the corresponding second velocity components, the values are $2.18 \pm 0.02$ and $1.18 \pm 0.03 \mathrm{~K} \mathrm{~km} \mathrm{~s}^{-1}$, respectively). For this calculation, the excitation temperature was assumed to be $5 \mathrm{~K}$ for both SMM 4 and Ori B9 N (and their second velocity components). This assumption is similar to the values calculated from Eq. (1) in all the other cases except the principal velocity component towards SMM 4, where $T_{\text {ex }}$ might be $>5 \mathrm{~K}$ (see Col. (7) of Table 2). Similarly, $N(2,2)$ (needed in the calculation of $T_{\text {rot }}$ using Eq. (2)) was calculated by assuming that $\tau \ll 1$, and using the integrated intensity of the main $(2,2)$ hyperfine complex, which accounts for $R_{i}=79.63 \%$ of the total line strength. In this case, we can write

$$
\begin{aligned}
& N(2,2)=\frac{3 k_{\mathrm{B}} \epsilon_{0}}{2 \pi^{2} \mu^{2} v_{22}} \frac{J(J+1)}{K^{2}} \frac{\mathrm{e}^{T_{22} / T_{\mathrm{ex}}}+1}{1-\frac{F\left(T_{\mathrm{bg}}\right)}{F\left(T_{\mathrm{ex}}\right)}} \frac{1}{R_{i}} \int T_{\mathrm{MB}}(2,2 ; \mathrm{m}) \mathrm{d} v \\
& =4.85 \times 10^{12} \frac{\mathrm{e}^{1.14 / T_{\mathrm{ex}}}+1}{1-\frac{F\left(T_{\mathrm{g} g}\right)}{F\left(T_{\mathrm{ex}}\right)}} \frac{1}{R_{i}} \int T_{\mathrm{MB}}(2,2 ; \mathrm{m}) \mathrm{d} v\left[\mathrm{~K} \mathrm{~km} \mathrm{~s}^{-1}\right] \mathrm{cm}^{-2},(7)
\end{aligned}
$$



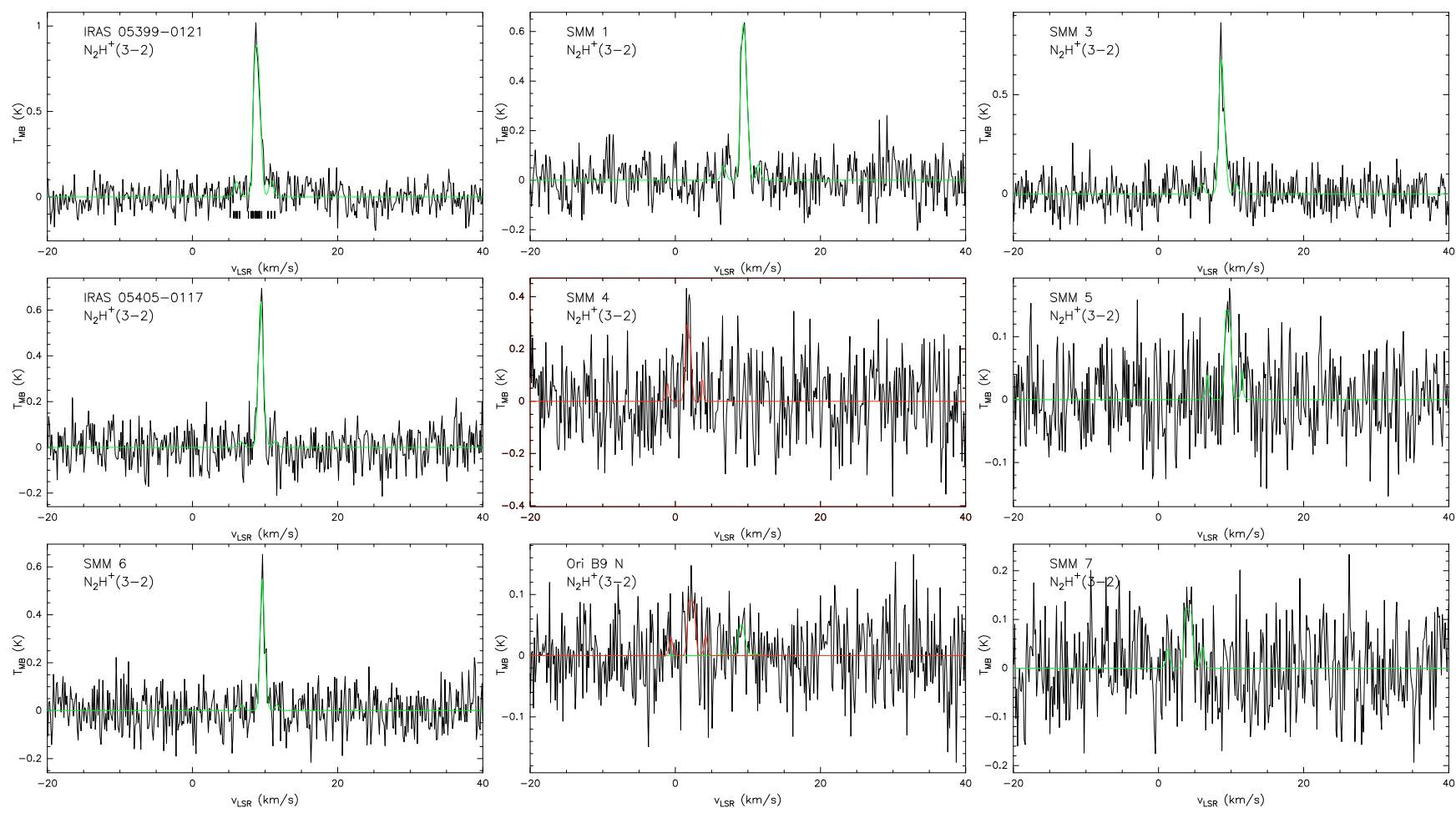

Fig. 3. $\mathrm{N}_{2} \mathrm{H}^{+}$(3-2) spectra measured from the pre- and protostellar cores in Orion B9. Spectra are overlaid with the 38 component hyperfine structure fits. Hyperfine fits to the second velocity components are indicated by red lines. Note that only the second velocity component is detected towards SMM 4. The relative velocity of each individual hyperfine component is labelled with a short bar on the spectrum towards IRAS 053990121 (top left panel). The temperature scale is in $T_{\mathrm{MB}}$.

where $v_{22}$ is the $(2,2)$ line frequency, and $T_{22}=h v_{22} / k_{\mathrm{B}}$. The integrated intensities of the $(2,2)$ main line for SMM 4 and Ori B $9 \mathrm{~N}$ are $0.21 \pm 0.01$ and $0.05 \pm 0.01 \mathrm{~K} \mathrm{~km} \mathrm{~s}^{-1}$, respectively $\left(0.15 \pm 0.01\right.$ and $0.21 \pm 0.03 \mathrm{~K} \mathrm{~km} \mathrm{~s}^{-1}$ for the corresponding second velocity components). The uncertainties associated with $N(1,1)$ and $N(2,2)$ were propagated from the formal errors in the integrated intensities.

The total $\mathrm{NH}_{3}$ column density, $N\left(\mathrm{NH}_{3}\right)$, was calculated by scaling the column density in the $(1,1)$ state by the ratio $N\left(\mathrm{NH}_{3}\right) / N(1,1)$ obtained from the partition function $Z$. This yields approximately (e.g., Rosolowsky et al. 2008; Busquet et al. 2009)

$N\left(\mathrm{NH}_{3}\right)=N(1,1)\left(\frac{1}{3} \mathrm{e}^{\frac{23.4}{T_{\text {rot }}}}+1+\frac{5}{3} \mathrm{e}^{-\frac{41.5}{T_{\text {rot }}}}+\frac{14}{3} \mathrm{e}^{-\frac{101.2}{T_{\text {rot }}}}\right)$.

The uncertainty in $N\left(\mathrm{NH}_{3}\right)$ was calculated by propagating the errors associated with $T_{\mathrm{ex}}, \Delta v, \tau_{\mathrm{m}}$, and $T_{\text {rot }}$. The values of $N\left(\mathrm{NH}_{3}\right)$ calculated by substituting $N(1,1)$ from Eq. (6) into Eq. (8) are mostly in good agreement, within the errors, with those resulting from Eqs. (5) and (8). In the subsequent analysis, we only use $\mathrm{NH}_{3}$ column densities derived for each source from Eqs. (5) and (8) to keep the data set homogeneous (see Col. (4) of Table 4).

\subsubsection{Fractional $\mathrm{NH}_{3}$ abundance}

The fractional $\mathrm{NH}_{3}$ abundance was calculated by dividing the total $\mathrm{NH}_{3}$ column density by the $\mathrm{H}_{2}$ column density, i.e., $x\left(\mathrm{NH}_{3}\right)=$ $N\left(\mathrm{NH}_{3}\right) / N\left(\mathrm{H}_{2}\right)$. The $\mathrm{H}_{2}$ column densities were determined from the submm dust continuum emission (Paper I; Eq. (3) therein), using the kinetic temperatures derived in the present paper and assuming that $T_{\text {kin }}=T_{\text {dust }}$ (see also Sect. 4.3). We smoothed the LABOCA $870 \mu \mathrm{m}$ map to correspond the $40^{\prime \prime}$ resolution of the Effelsberg $\mathrm{NH}_{3}$ observations.

\subsubsection{Non-thermal velocity dispersion and the level of internal turbulence}

The measured $\mathrm{NH}_{3}(1,1)$ linewidths were used to calculate the non-thermal portion of the line-of-sight velocity dispersion (averaged over a $\sim 40^{\prime \prime}$ beam), and the level of internal turbulence. The observed velocity dispersion is related to the FWHM linewidth as $\sigma_{\mathrm{obs}}=\Delta v / \sqrt{8 \ln 2}$. The non-thermal velocity dispersion can then be calculated as follows:

$\sigma_{\mathrm{NT}}=\sqrt{\sigma_{\mathrm{obs}}^{2}-\frac{k_{\mathrm{B}} T_{\mathrm{kin}}}{\mu_{\mathrm{mol}} m_{\mathrm{H}}}}$,

where $\mu_{\mathrm{mol}}$ is the mass of the emitting molecule in units of atomic mass number ( 17 for $\mathrm{NH}_{3}$ ), and $m_{\mathrm{H}}$ is the mass of the hydrogen atom. Furthermore, the level of internal turbulence is given by $f_{\text {turb }}=\sigma_{\mathrm{NT}} / c_{\mathrm{s}}$, where $c_{\mathrm{s}}$ is the one-dimensional isothermal sound speed $\left(0.19 \mathrm{~km} \mathrm{~s}^{-1}\right.$ in a $10 \mathrm{~K} \mathrm{H}_{2}$ gas with $\left.10 \% \mathrm{He}\right)$. The errors in $\sigma_{\mathrm{NT}}$ and $f_{\text {turb }}$ were derived by propagating the errors in $\Delta v$ and $T_{\text {kin. }}$.

\section{2. $\mathrm{N}_{2} \mathrm{H}^{+}$analysis}

In this subsection, we derive physical parameters from $\mathrm{N}_{2} \mathrm{H}^{+}$data. The obtained results are given in Col. (6) of Table 3 $\left(T_{\mathrm{ex}}\right)$, and in Table 5.

\subsection{1. $T_{\text {ex }}, \sigma_{\mathrm{NT}}$, and $f_{\text {turb }}$}

The excitation temperature of the $\mathrm{N}_{2} \mathrm{H}^{+}(3-2)$ transition was calculated as in the case of $\mathrm{NH}_{3}(1,1)$ (see Eq. (1)). The optical thickness, and thus $T_{\text {ex }}$, could be determined only for three sources: IRAS 05399-0121, and SMM 1 and 3. For the rest of the sources, it was assumed that $T_{\mathrm{ex}}=5 \mathrm{~K}$, and $\tau_{\text {tot }}$ was then 
Table 3. $\mathrm{N}_{2} \mathrm{H}^{+}(3-2)$ line parameters.

\begin{tabular}{cccccc}
\hline \hline & $\begin{array}{c}v_{\mathrm{LSR}} \\
{\left[\mathrm{km} \mathrm{s}^{-1}\right]}\end{array}$ & $\begin{array}{c}\Delta v \\
{\left[\mathrm{~km} \mathrm{~s}^{-1}\right]}\end{array}$ & $\begin{array}{c}T_{\mathrm{MB}}{ }^{a} \\
{[\mathrm{~K}]}\end{array}$ & $\tau_{\text {tot }}{ }^{b}$ & $\begin{array}{c}T_{\mathrm{ex}} \\
{[\mathrm{K}]}\end{array}$ \\
\hline Source & $8.73 \pm 0.02$ & $0.67 \pm 0.07$ & $0.92 \pm 0.04$ & $3.08 \pm 1.10$ & $5.1 \pm 0.3$ \\
\hline IRAS 05399-0121 & $9.30 \pm 0.03$ & $0.61 \pm 0.09$ & $0.66 \pm 0.07$ & $2.77 \pm 1.48$ & $4.6 \pm 0.3$ \\
SMM 1 & $8.57 \pm 0.03$ & $0.71 \pm 0.02$ & $0.65 \pm 0.08$ & $0.92 \pm 0.19$ & $5.5 \pm 0.5$ \\
SMM 3 & $9.30 \pm 0.03$ & $0.68 \pm 0.06$ & $0.66 \pm 0.07$ & $1.48 \pm 0.34^{c}$ & $5.0^{d}$ \\
IRAS 05405-0117 & - & - & - & - & - \\
SMM 4 & $1.60 \pm 0.10$ & $0.44 \pm 0.25$ & $0.35 \pm 0.13$ & $0.54 \pm 0.26^{c}$ & $5.0^{d}$ \\
SMM 4 (2nd v-comp.) & $9.43 \pm 0.07$ & $0.46 \pm 0.13$ & $0.17 \pm 0.06$ & $0.23 \pm 0.09^{c}$ & $5.0^{d}$ \\
SMM 5 & $9.52 \pm 0.03$ & $0.58 \pm 0.09$ & $0.53 \pm 0.08$ & $0.99 \pm 0.24^{c}$ & $5.0^{d}$ \\
SMM 6 & $8.78 \pm 0.13$ & $0.22 \pm 0.32^{f}$ & $<0.07$ & $<0.10^{c}$ & $5.0^{d}$ \\
Ori B9 N & $2.07 \pm 0.10$ & $0.43 \pm 0.22$ & $0.10 \pm 0.05$ & $0.13 \pm 0.07^{c}$ & $5.0^{d}$ \\
Ori B9 N (2nd v-comp.) & $3.96 \pm 0.14$ & $0.66 \pm 0.22$ & $0.13 \pm 0.08$ & $0.17 \pm 0.11^{c}$ & $5.0^{d}$ \\
SMM 7 & & & & &
\end{tabular}

Notes. ${ }^{(a)}$ The error in $T_{\mathrm{MB}}$ is the $1 \sigma$ rms noise error. ${ }^{(b)} \tau_{\text {tot }}$ is the total optical thickness of the hyperfine multiplet. ${ }^{(c)} \tau_{\text {tot }}$ could not be determined through fitting the hyperfine structure. Instead, $\tau_{\text {tot }}$ was calculated by assuming $T_{\mathrm{ex}}=5.0 \mathrm{~K}$, and taking into account that the line intensity in Col. (4) accounts for $92.6 \%$ of the total line strength. ${ }^{(d)}$ Assumed value. ${ }^{(e)}$ The principal velocity component was not detected. ${ }^{(f)}$ The associated error is larger than the value.

Table 4. Parameters derived from $\mathrm{NH}_{3}$ data.

\begin{tabular}{ccccccc}
\hline \hline & $T_{\text {rot }}$ & $\begin{array}{c}T_{\text {kin }} \\
{[\mathrm{K}]}\end{array}$ & $\begin{array}{c}N\left(\mathrm{NH}_{3}\right) \\
{\left[10^{14} \mathrm{~cm}^{-2}\right]}\end{array}$ & $\begin{array}{c}x\left(\mathrm{NH}_{3}\right) \\
{\left[10^{-8}\right]}\end{array}$ & $\begin{array}{c}\sigma_{\mathrm{NT}} \\
{\left[\mathrm{km} \mathrm{s}^{-1}\right]}\end{array}$ & $f_{\text {turb }}$ \\
\hline Source & $12.5 \pm 1.4$ & $13.5 \pm 1.6$ & $3.2 \pm 0.6$ & $1.5 \pm 0.4$ & $0.24 \pm 0.01$ & $1.1 \pm 0.1$ \\
\hline IRAS 05399-0121 & $11.2 \pm 0.8$ & $11.9 \pm 0.9$ & $6.2 \pm 0.9$ & $3.5 \pm 0.7$ & $0.27 \pm 0.004$ & $1.3 \pm 0.1$ \\
SMM 1 & $11.1 \pm 1.6$ & $11.8 \pm 1.8$ & $2.6 \pm 0.7$ & $4.1 \pm 0.7$ & $0.12 \pm 0.01$ & $0.6 \pm 0.1$ \\
SMM 2 & $10.7 \pm 0.7$ & $11.3 \pm 0.8$ & $5.6 \pm 0.8$ & $1.9 \pm 0.4$ & $0.14 \pm 0.003$ & $0.7 \pm 0.04$ \\
SMM 3 & $10.7 \pm 0.6$ & $11.3 \pm 0.6$ & $10.0 \pm 1.3$ & $9.8 \pm 1.6$ & $0.11 \pm 0.002$ & $0.6 \pm 0.02$ \\
IRAS 05405-0117 & $12.8 \pm 0.6$ & $13.9 \pm 0.8$ & $3.4 \pm 0.4$ & $3.8 \pm 0.6$ & $0.12 \pm 0.005$ & $0.5 \pm 0.03$ \\
SMM 4 & $10.2 \pm 0.9^{a}$ & $10.7 \pm 1.1^{a}$ & $8.8 \pm 1.4^{b}$ & & & \\
SMM 4 (2nd v-comp.) & $10.0 \pm 1.2$ & $10.4 \pm 1.4$ & $6.8 \pm 1.6$ & $4.5 \pm 1.6$ & $0.29 \pm 0.01$ & $1.5 \pm 0.1$ \\
& $9.7 \pm 1.0^{a}$ & $10.2 \pm 1.1^{a}$ & $8.2 \pm 1.6^{b}$ & & & \\
SMM 5 & $10.7 \pm 0.6$ & $11.3 \pm 0.7$ & $5.9 \pm 0.7$ & $8.0 \pm 1.3$ & $0.13 \pm 0.002$ & $0.6 \pm 0.02$ \\
SMM 6 & $10.4 \pm 0.3$ & $11.0 \pm 0.4$ & $9.9 \pm 0.9$ & $7.2 \pm 0.8$ & $0.10 \pm 0.001$ & $0.5 \pm 0.01$ \\
Ori B9 N & $12.4 \pm 1.1$ & $13.4 \pm 1.3$ & $2.0 \pm 0.4$ & $3.8 \pm 0.9$ & $0.15 \pm 0.01$ & $0.7 \pm 0.04$ \\
& $9.7 \pm 3.6^{a}$ & $10.2 \pm 4.0^{a}$ & $2.8 \pm 1.9^{b}$ & & & \\
Ori B9 N (2nd v-comp.) & $12.5 \pm 2.1$ & $13.6 \pm 2.5$ & $2.5 \pm 0.8$ & $5.0 \pm 2.3$ & $0.63 \pm 0.02$ & $2.9 \pm 0.3$ \\
& $12.5 \pm 1.7^{a}$ & $13.6 \pm 1.9^{a}$ & $3.0 \pm 0.5^{b}$ & & & \\
SMM 7 & $9.0 \pm 1.0$ & $9.4 \pm 1.1$ & $8.0 \pm 2.0$ & $5.3 \pm 1.9$ & $0.25 \pm 0.01$ & $1.3 \pm 0.1$ \\
IRAS 05413-0104 & $12.3 \pm 1.0$ & $13.4 \pm 1.2$ & $3.7 \pm 0.5$ & $3.4 \pm 0.7$ & $0.18 \pm 0.004$ & $0.8 \pm 0.04$ \\
\hline
\end{tabular}

Notes. ${ }^{(a)}$ Calculated by using the Eq. (2). ${ }^{(b)}$ Calculated by using Eqs. (6) and (8).

estimated using this value. The value $T_{\mathrm{ex}}=5 \mathrm{~K}$ is expected to be a reasonable choice because for the above three sources $T_{\text {ex }}$ is around 5 kelvins.

The values of $\sigma_{\mathrm{NT}}$ and $f_{\text {turb }}$ were calculated as in the case of $\mathrm{NH}_{3}(1,1)$ (see Eq. (9)), by using the values of $T_{\text {kin }}$ from $\mathrm{NH}_{3}$ measurements. For $\mathrm{N}_{2} \mathrm{H}^{+}, \mu_{\mathrm{mol}}$ is 29 .

\subsection{2. $\mathrm{N}_{2} \mathrm{H}^{+}$column density and fractional abundance}

The $\mathrm{N}_{2} \mathrm{H}^{+}$column density was calculated using the formula (see, e.g., Paper I)

$$
\begin{aligned}
& N\left(\mathrm{~N}_{2} \mathrm{H}^{+}\right)=\frac{3 h \epsilon_{0}}{2 \pi^{2} \mu^{2}} \frac{\sqrt{\pi}}{2 \sqrt{\ln 2}} \frac{1}{J_{\mathrm{u}}} \mathrm{e}^{E_{\mathrm{u}} / k_{\mathrm{B}} T_{\text {ex }}} F\left(T_{\text {ex }}\right) Z\left(T_{\text {ex }}\right) \Delta v \tau_{\text {tot }} \\
& =2.46 \times 10^{11} \mathrm{e}^{26.83 / T_{\text {ex }}} F\left(T_{\text {ex }}\right) Z\left(T_{\text {ex }}\right) \Delta v\left[\mathrm{~km} \mathrm{~s}^{-1}\right] \tau_{\text {tot }} \mathrm{cm}^{-2},
\end{aligned}
$$

where $\mu$ is $3.4 \mathrm{D}$, the upper rotational level number $J_{\mathrm{u}}=3, E_{\mathrm{u}}=$ $h B J_{\mathrm{u}}\left(J_{\mathrm{u}}+1\right)$ is the energy of the upper transition state, and $B=$ $46586.8713 \mathrm{MHz}$ is the rotational constant (Pagani et al. 2009).
The rotational partition function was approximated by $Z\left(T_{\mathrm{ex}}\right)=$ $\left(\frac{k_{\mathrm{B}} T_{\mathrm{ex}}}{h B}+\frac{1}{3}\right)$.

In those cases where the line was optically thin (i.e., SMM 5, Ori B9 $\mathrm{N}$ and its second velocity component, and SMM 7), the $\mathrm{N}_{2} \mathrm{H}^{+}$column density was also calculated from the integrated intensity. The integrated intensities obtained from Gaussian fits are $0.15 \pm 0.03,0.07 \pm 0.16(0.12 \pm 0.04)$, and $0.22 \pm 0.07 \mathrm{~K} \mathrm{~km} \mathrm{~s}^{-1}$ for SMM 5, Ori B9 N (and its second velocity component), and SMM 7, respectively. Note that the error is larger than the value for Ori B9 N. By combining Eqs. (4) and (6) of Paper I, we get the following formula for the $N\left(\mathrm{~N}_{2} \mathrm{H}^{+}\right)$as a function of integrated intensity:

$$
\begin{aligned}
& N\left(\mathrm{~N}_{2} \mathrm{H}^{+}\right)=\frac{3 \epsilon_{0} k_{\mathrm{B}}}{2 \pi^{2} \mu^{2} v} \frac{1}{J_{\mathrm{u}}} \mathrm{e}^{E_{\mathrm{u}} / k_{\mathrm{B}} T_{\mathrm{ex}}} Z\left(T_{\mathrm{ex}}\right) \frac{1}{1-\frac{F\left(T_{\mathrm{bg}}\right)}{F\left(T_{\mathrm{ex}}\right)}} \int T_{\mathrm{MB}} \mathrm{d} v \\
& =1.72 \times 10^{10} Z\left(T_{\mathrm{ex}}\right) \frac{\mathrm{e}^{26.83 / T_{\mathrm{ex}}}}{1-\frac{F\left(T_{\mathrm{bg}}\right)}{F\left(T_{\mathrm{ex}}\right)}} \int T_{\mathrm{MB}} \mathrm{d} v\left[\mathrm{~K} \mathrm{~km} \mathrm{~s}^{-1}\right] \mathrm{cm}^{-2}
\end{aligned}
$$

When applying Eq. (11), it was taken into account that the detectable emission feature with 24 hyperfine components 

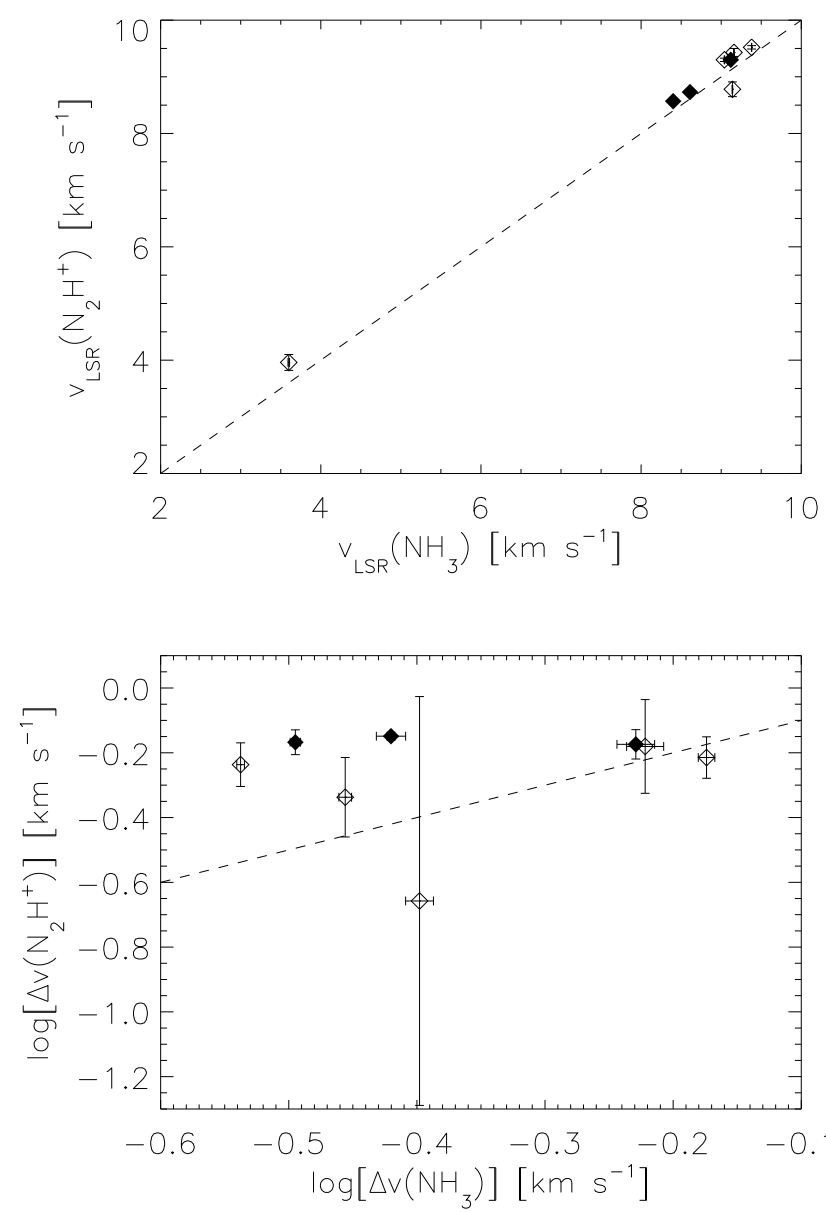

Fig. 4. Top: the centroid velocity of the $\mathrm{N}_{2} \mathrm{H}^{+}(3-2)$ emission versus the velocity of the $\mathrm{NH}_{3}(1,1)$ emission. Bottom: Log-log plot of the $\mathrm{N}_{2} \mathrm{H}^{+}(3-2)$ linewidth versus the $\mathrm{NH}_{3}(1,1)$ linewidth. Prestellar cores are indicated by open diamonds and protostellar cores are marked by filled diamonds. In both plots, the dashed line shows the equality.

contains $92.6 \%$ of the total line strength. The values of $N\left(\mathrm{~N}_{2} \mathrm{H}^{+}\right)$ calculated from Eqs. (10) and (11) are similar to each other within the errors (see Table 5).

The fractional $\mathrm{N}_{2} \mathrm{H}^{+}$abundance was calculated in a similar manner as for $\mathrm{NH}_{3}$. In this case, the LABOCA map was smoothed to the resolution of the $\mathrm{N}_{2} \mathrm{H}^{+}$observations (22'. 3).

\subsection{Revision of core properties presented in Paper I}

In Paper I, we assumed that the dust temperature is $T_{\text {dust }}=10 \mathrm{~K}$ for the starless cores, whereas for protostellar cores we used the temperatures derived from the spectral energy distribution (SED) fits (Table 6 in Paper I). By using the gas kinetic temperatures determined in the present paper, we recalculated several parameters presented in Paper I, by assuming that $T_{\text {kin }}=T_{\text {dust }}$. These include the core mass, $M, \mathrm{H}_{2}$ column density, $N\left(\mathrm{H}_{2}\right)$, and the average $\mathrm{H}_{2}$ number density, $\left\langle n\left(\mathrm{H}_{2}\right)\right\rangle$. In the present paper, we have also amended some formula used in the derivation of the physical parameters: in the calculation of $\left\langle n\left(\mathrm{H}_{2}\right)\right\rangle$, we have used the effective radius $R_{\text {eff }}=\sqrt{A / \pi}$ (instead of the $F W H M$ radius used in Paper I), and the value $2.8 m_{\mathrm{H}}$ for the mean molecular weight per $\mathrm{H}_{2}$ molecule (instead of $2.33 m_{\mathrm{H}}$ which is the mean particle weight in an $\mathrm{H}_{2}+10 \%$ He mixture). The former change is motivated by the fact that the flux densities used to calculate the core masses refer to their projected areas, $A$. The results of these calculations are presented in Table 6 . The revised values of $M$ and $N\left(\mathrm{H}_{2}\right)$, are, on average, about $11 \%$ and $9 \%$ higher than those reported in Paper I. On the other hand, the new average densities, $\left\langle n\left(\mathrm{H}_{2}\right)\right\rangle$, are only about $1 / 5$ of those calculated in Paper I. Note that the total mass of the cores is still $\sim 50 \mathrm{M}_{\odot}$, i.e., about $4 \%$ of the total mass in the region (Paper I).

In Paper I, we compared the core mass distributions in Orion B9 and Orion B North studied by Nutter \& WardThompson (2007) (Sect. 5.2 and Fig. 7 in Paper I). The core mass functions (CMFs) were constructed by removing the Class I protostellar cores from the samples (i.e., IRAS 05399-0121 was removed from the Orion B9 core sample). We performed a twosample Kolmogorov-Smirnov (K-S) test between the two CMFs to examine if they represent the subsamples of the same underlying parent distribution. For this purpose, the masses were scaled to compensate for the different assumptions about the distance, dust temperature, and opacity. We found a very high likelihood of $\sim 95 \%$ for the null hypothesis that the two CMFs are drawn from the same parent distribution (i.e., the probability that the two samples do not have the same parent distribution is $1-0.95=0.05$ ). We repeated this analysis by using the updated masses listed in Col. (3) of Table 6. In this case, a K-S test yielded a probability of $\sim 100 \%$, strengthening the possibility that the core masses in these two different parts of Orion B are drawn from the same distribution.

\subsection{Virial masses}

In order to study the stability of the cores, we calculated their virial masses using the following formula where the effects of external pressure and magnetic field are ignored:

$M_{\mathrm{vir}}=\frac{5}{8 \ln 2} \frac{R_{\mathrm{eff}} \Delta v_{\mathrm{ave}}^{2}}{a G}$,

where $G$ is the gravitational constant, and $\Delta v_{\text {ave }}$ is the width of the spectral line emitted by the molecule of mean mass $\mu=2.33$. The parameter $a=(1-p / 3) /(1-2 p / 5)$, where $p$ is the powerlaw index of the density profile $\left(n(r) \propto r^{-p}\right)$, is a correction for deviations from constant density. For starless cores we used the value $p=1.0$, whereas for protostellar cores we set $p=1.5$ (see Paper I). The value of $M_{\text {vir }}$ calculated with $p=1.0$ is about $13 \%$ higher compared to that calculated with the value $p=1.5$. As a function of the observed linewidth, $\Delta v_{\text {obs }}$ (Table 2, Col. (4)), $\Delta v_{\text {ave }}$ is given by

$$
\Delta v_{\mathrm{ave}}^{2}=\Delta v_{\mathrm{T}}^{2}+\Delta v_{\mathrm{NT}}^{2}=\Delta v_{\mathrm{obs}}^{2}+8 \ln 2 \times \frac{k_{\mathrm{B}} T_{\mathrm{kin}}}{m_{\mathrm{H}}}\left(\frac{1}{\mu}-\frac{1}{\mu_{\mathrm{mol}}}\right) \text {. }
$$

The virial masses are listed in Col. (4) of Table 6. The associated error was propagated from those of $\Delta v_{\text {obs }}$ and $T_{\text {kin }}$.

The virial parameters of the cores were calculated following Bertoldi \& McKee (1992), i.e., $\alpha_{\text {vir }}=M_{\text {vir }} / M$. The uncertainty was derived by propagating the errors in both mass estimates. The values are given in Col. (5) of Table 6. Note that $\alpha_{\text {vir }}=1$ corresponds to the virial equilibrium, $2\langle T\rangle=-\langle U\rangle$, where $T$ and $U$ are the internal kinetic and gravitational energies, respectively. The value $\alpha_{\text {vir }}=2$ corresponds to the self-gravitating limit defined by $\langle T\rangle=-\langle U\rangle$.

\section{Discussion}

\subsection{The gas kinetic temperature in dense cores in Orion $B$}

The gas kinetic temperatures within the cores in Orion B9 are in the range $\sim 9.4-13.9 \mathrm{~K}$, with a mean value of $12.0 \pm 0.4 \mathrm{~K}$ (the 
Table 5. Parameters derived from $\mathrm{N}_{2} \mathrm{H}^{+}(3-2)$ data.

\begin{tabular}{cccccc}
\hline \hline Source & $\begin{array}{c}N\left(\mathrm{~N}_{2} \mathrm{H}^{+}\right) \\
{\left[10^{13} \mathrm{~cm}^{-2}\right]}\end{array}$ & $\begin{array}{c}x\left(\mathrm{~N}_{2} \mathrm{H}^{+}\right) \\
{\left[10^{-10}\right]}\end{array}$ & $N\left(\mathrm{NH}_{3}\right) / N\left(\mathrm{~N}_{2} \mathrm{H}^{+}\right)$ & $\begin{array}{c}\sigma_{\mathrm{NT}} \\
{\left[\mathrm{km} \mathrm{s}^{-1}\right]}\end{array}$ & $f_{\text {turb }}$ \\
\hline IRAS 05399-0121 & $2.0 \pm 0.7$ & $4.3 \pm 1.8$ & $16 \pm 7$ & $0.28 \pm 0.03$ & $1.3 \pm 0.2$ \\
SMM 1 & $1.9 \pm 1.1$ & $5.9 \pm 3.4$ & $32 \pm 18$ & $0.25 \pm 0.04$ & $1.2 \pm 0.2$ \\
SMM 3 & $0.6 \pm 0.1$ & $0.8 \pm 0.2$ & $99 \pm 25$ & $0.30 \pm 0.01$ & $1.5 \pm 0.1$ \\
IRAS 05405-0117 & $1.0 \pm 0.2^{a}$ & $5.5 \pm 1.5$ & $100 \pm 28$ & $0.28 \pm 0.03$ & $1.4 \pm 0.1$ \\
SMM 4 & - & - & - & - & - \\
SMM 4 (2nd v-comp.) & $0.2 \pm 0.2$ & $0.8 \pm 0.6$ & $288 \pm 225$ & $0.18 \pm 0.11$ & $0.9 \pm 0.6$ \\
SMM 5 & $0.1 \pm 0.1$ & $0.7 \pm 0.4$ & $562 \pm 279$ & $0.19 \pm 0.06$ & $0.9 \pm 0.3$ \\
& $0.2 \pm 0.03^{c}$ & $1.2 \pm 0.2^{c}$ & $275 \pm 54^{c}$ & & \\
SMM 6 & $0.6 \pm 0.2$ & $2.1 \pm 0.6$ & $194 \pm 80$ & $0.24 \pm 0.04$ & $1.2 \pm 0.2$ \\
Ori B9 N & $0.02 \pm 0.03$ & $0.2 \pm 0.3$ & $916 \pm 1345^{d}$ & $0.07 \pm 0.18^{d}$ & $0.3 \pm 0.8$ \\
& $0.1 \pm 0.2^{c, d}$ & $0.8 \pm 0.1^{c}$ & $280 \pm 190^{c}$ & & \\
Ori B9 N (2nd v-comp.) & $0.1 \pm 0.04$ & $0.6 \pm 0.5$ & $250 \pm 90$ & $0.17 \pm 0.10$ & $0.8 \pm 0.5$ \\
& $0.1 \pm 0.05^{c}$ & $1.1 \pm 0.4^{c}$ & $250 \pm 154^{c}$ & & \\
SMM 7 & $0.1 \pm 0.1$ & $0.3 \pm 0.2$ & $718 \pm 553$ & $0.28 \pm 0.10$ & $1.5 \pm 0.5$ \\
& $0.3 \pm 0.1^{c}$ & $0.7 \pm 0.3^{c}$ & $267 \pm 91^{c}$ & & \\
\hline
\end{tabular}

Notes. ${ }^{(a)}$ In Paper I, we derived the value $N\left(\mathrm{~N}_{2} \mathrm{H}^{+}\right) \approx 0.91 \pm 0.01 \times 10^{13} \mathrm{~cm}^{-2}$ from observations of $\mathrm{N}_{2} \mathrm{H}^{+}(1-0)$ emission, which is in good agreement with the present value. ${ }^{(b)}$ The principal velocity component was not detected. ${ }^{(c)}$ Calculated by using the optically thin approximation. (d) The associated error is larger than the value.

Table 6. Revised core parameters presented in Paper I.

\begin{tabular}{ccccccc}
\hline \hline Source & $\begin{array}{c}R_{\mathrm{eff}} \\
{[\mathrm{pc}]}\end{array}$ & $\begin{array}{c}M^{a} \\
{\left[M_{\odot}\right]}\end{array}$ & $\begin{array}{c}M_{\mathrm{vir}}{ }^{b} \\
{\left[M_{\odot}\right]}\end{array}$ & $\alpha_{\mathrm{vir}}{ }^{b}$ & $\begin{array}{c}N\left(\mathrm{H}_{2}\right)^{a} \\
{\left[10^{22} \mathrm{~cm}^{-2}\right]}\end{array}$ & $\begin{array}{c}\left\langle n\left(\mathrm{H}_{2}\right)\right\rangle^{a} \\
{\left[10^{4} \mathrm{~cm}^{-3}\right]}\end{array}$ \\
\hline IRAS 05399-0121 & 0.08 & $6.1 \pm 1.4$ & $7.8 \pm 0.5$ & $1.3 \pm 0.3$ & $4.2 \pm 0.9$ & $5.5 \pm 1.3$ \\
SMM 1 & 0.09 & $8.4 \pm 1.5$ & $11.1 \pm 0.3$ & $1.3 \pm 0.2$ & $2.8 \pm 0.4$ & $5.3 \pm 0.9$ \\
SMM 2 & 0.08 & $2.0 \pm 0.6$ & $4.8 \pm 0.5$ & $2.4 \pm 0.8$ & $1.4 \pm 0.4$ & $1.8 \pm 0.5$ \\
SMM 3 & 0.07 & $7.8 \pm 1.6$ & $4.0 \pm 0.2$ & $0.5 \pm 0.1$ & $8.4 \pm 1.1$ & $10.5 \pm 2.1$ \\
IRAS 05405-0117 & 0.07 & $2.8 \pm 0.4$ & $3.5 \pm 0.1$ & $1.2 \pm 0.2$ & $1.4 \pm 0.1$ & $3.8 \pm 0.5$ \\
SMM 4 & 0.07 & $2.8 \pm 0.3$ & $4.1 \pm 0.2$ & $1.5 \pm 0.2$ & $1.4 \pm 0.1$ & $3.8 \pm 0.4$ \\
SMM 4 (2nd v-comp.) & & & & & $2.4 \pm 0.7$ & \\
SMM 5 & 0.07 & $1.9 \pm 0.4$ & $4.2 \pm 0.2$ & $2.2 \pm 0.5$ & $1.2 \pm 0.1$ & $2.5 \pm 0.5$ \\
SMM 6 & 0.12 & $8.2 \pm 1.1$ & $6.2 \pm 0.2$ & $0.8 \pm 0.1$ & $2.0 \pm 0.1$ & $2.2 \pm 0.3$ \\
Ori B9 N & 0.08 & $2.3 \pm 0.4$ & $5.8 \pm 0.4$ & $2.5 \pm 0.5$ & $0.9 \pm 0.1$ & $2.1 \pm 0.4$ \\
Ori B9 N (2nd v-comp.) & & & & & $0.9 \pm 0.1$ & \\
SMM 7 & 0.07 & $3.6 \pm 1.0$ & $6.9 \pm 0.4$ & $1.9 \pm 0.6$ & $3.4 \pm 0.9$ & $4.8 \pm 1.3$ \\
IRAS 05413-0104 & 0.06 & $2.0 \pm 0.6$ & $4.4 \pm 0.2$ & $2.2 \pm 0.7$ & $3.6 \pm 0.6$ & $4.3 \pm 1.3$ \\
\hline
\end{tabular}

Notes. ${ }^{(a)}$ The masses and $\mathrm{H}_{2}$ column and number densities were calculated using the values of $T_{\text {kin }}\left(=T_{\text {dust }}\right){ }^{(b)}$ The table also lists the core virial masses and virial parameters (Cols. (4) and (5)).

quoted error is the standard deviation of the mean). The highest temperatures (13.4-13.9 K) are found towards IRAS 053990121, SMM 4, Ori B9 N, and IRAS 05413-0104. There is, however, no such tendency that the warmest cores should be protostellar. For example, the Class 0 sources SMM 3 and IRAS 05405-0117 have $T_{\text {kin }}$ values at the low end in the sample (11.3 K for both sources). This is consistent with the notion that embedded low-mass protostars do not heat significantly the parent cores, but the heating is localised in their immediate vicinity ( 100 AU; Friesen et al. 2010 and references therein).

Recently, based on the Nobeyama 45-m observations, Ikeda et al. (2009) derived the $\mathrm{NH}_{3}$ rotational temperatures towards 144 positions in Orion B. The cores No. 55-66 in the Ikeda et al. (2009) sample lie in the Orion B9 region. For these latter cores, they found the values in the range $T_{\text {rot }}=10.1-16.1 \mathrm{~K}$. By using the relationship (4), this corresponds to the $T_{\text {kin }}$ values $10.6-18.5 \mathrm{~K}$, with the mean value $16.4 \mathrm{~K}$. This is clearly higher than the mean $T_{\text {kin }}$ of the present study. The difference is likely to be caused by the larger beamsize used by Ikeda et al. $(\sim 1$ ! 3). We note that the highest value of $T_{\text {kin }}=43.9 \mathrm{~K}$ in the Ikeda et al. (2009) sample was found in the NGC 2024 H II region, and the lowest values $(\lesssim 11 \mathrm{~K})$ were found in Orion B9 and other regions of relatively low star formation activity, i.e., outside the NGC 2023, 2024, 2068, and 2071 regions.

We note that in several previous submm surveys of Orion B dust temperatures around $20 \mathrm{~K}$ have been derived or assumed for dense cores (Johnstone et al. 2001, 2006; Nutter \& Ward-Thompson 2007). Together with the results of Ikeda et al. (2009) the present temperature determinations demonstrate that Orion B contains also several very cold cores resembling cores in nearby low-mass star forming regions (see, e.g., Rosolowsky et al. 2008; Schnee et al. 2009; Friesen et al. 2009 and references therein).

\subsection{Kinematics of the core gas}

The $\mathrm{NH}_{3}$ line profiles show that most cores have subsonic nonthermal motions $\left(f_{\text {turb }}<1\right)$. In two cores (SMM 1 and 7), $f_{\text {turb }}$ is higher than in the rest of the cores, and non-thermal motions appear to be slightly transonic $\left(f_{\text {turb }}=1.3<2\right)$. Recently, Friesen et al. (2009) found that the mean $f_{\text {turb }}$ value for dense cores in 


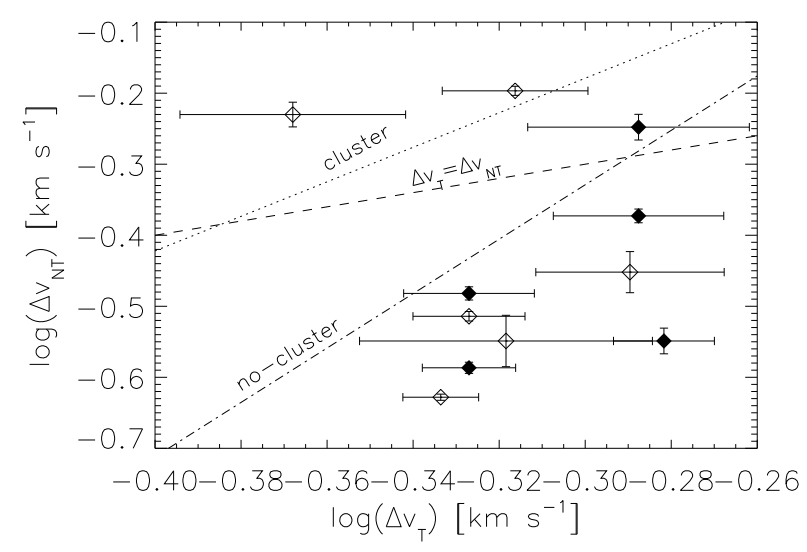

Fig. 5. Non-thermal versus thermal (for $\mathrm{H}_{2}$, i.e., the isothermal sound speed) linewidths in log-log scales. The dashed line indicates $\Delta v_{\mathrm{NT}}=$ $\Delta v_{\mathrm{T}}$. The dotted and dash-dotted lines show relationships found by Jijina et al. (1999) for $\mathrm{NH}_{3}$ cores with and without cluster association, respectively. Symbols have the same meaning as in Fig. 4.

Oph B is 1.5 , clearly larger than the corresponding value for our cores (a mean $f_{\text {turb }}$ and its standard deviation is $0.8 \pm 0.1$ ).

In Fig. 5, we plot the non-thermal linewidth against the thermal linewidth for the cores. Figure 5 shows that most of the cores in Orion B9 are quiescent, i.e., $\Delta v_{\mathrm{NT}}<\Delta v_{\mathrm{T}}$. Also shown in this figure are the $\Delta v_{\mathrm{NT}}-\Delta v_{\mathrm{T}}$ relationships found by Jijina et al. (1999) for $\mathrm{NH}_{3}$ cores in clusters and isolated regions. The two relationships suggest that non-thermal $\mathrm{NH}_{3}$ linewidths are larger for cores in clustered environments than in isolated cores. A majority of Orion B9 cores lie on the Jijina plot in the region characteristic of isolated cores.

Like in the case of the kinetic temperature, there is no clear difference in linewidths between starless and protostellar cores. Protostars are likely to be associated with outflows which could cause the linewidths to be larger, but evidence for outflows can only be found in IRAS 05399-0121 (HH 92; Bally et al. 2002) and IRAS 05413-0104 (HH 212; e.g., Lee et al. 2008). It is possible that the HH 92 outflow from IRAS 05399-0121 contribute to the relatively broad $\mathrm{NH}_{3}$ lines seen in the adjacent SMM 1 core which is oriented along the direction of the outflow.

In Fig. 6, we plot the $\mathrm{NH}_{3}(1,1)$ linewidth as a function of core effective radius. Also shown are the linewidth-size relation derived by Larson (1981), and the relationship recently found by Ikeda et al. (2009) for the $\mathrm{H}^{13} \mathrm{CO}^{+}$cores in Orion B. The Larson relation, thought to arise from the interstellar turbulence, was originally presented for the $3 \mathrm{D}$ velocity dispersion, $\sigma_{3 \mathrm{D}}$, and the maximum linear size of the source, $L$. In terms of our definitions, it can be written as $\Delta v\left[\mathrm{~km} \mathrm{~s}^{-1}\right]=$ $1.9\left(R_{\mathrm{eff}}[\mathrm{pc}]\right)^{0.38}$, where we have assumed that $L=2 R_{\mathrm{eff}}$. The linewidths of Orion B9 cores mostly deviate downward from the Larson relation, and the cores lie around the Ikeda et al. (2009) relationship, namely $\Delta v\left[\mathrm{~km} \mathrm{~s}^{-1}\right]=1.28\left(R_{\text {core }}[\mathrm{pc}]\right)^{0.38}$ (we remind the reader that the Ikeda et al. sample contains Orion B9 members). On the other hand, it is possible that the more tenuous gas in Orion B9 follows the Larson relation: the mean linewidth $\left(\sim 1.3 \mathrm{~km} \mathrm{~s}^{-1}\right)$ and half-maximum radius $(\sim 0.6 \mathrm{pc})$ measured from the ${ }^{13} \mathrm{CO}(1-0)$ emission towards the Orion B9 region by Caselli \& Myers (1995) are quite similar to those expected from the Larson relation. As pointed out by Maruta et al. (2010), this could be explained if the cores were formed in regions where supersonic turbulence was dissipated. Dissipation of turbulence could be due to shocks in the converging turbu-

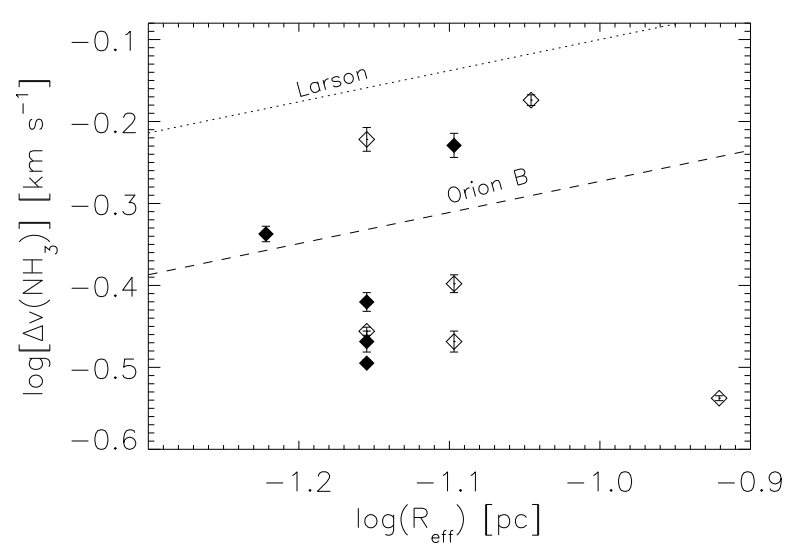

Fig. 6. Linewidth versus radius for the cores in Orion B9 in log-log scales. The Larson (1981) relation is shown by the dotted line. The dashed line indicates the relation derived by Ikeda et al. (2009) for the $\mathrm{H}^{13} \mathrm{CO}^{+}$cores in Orion B. Symbols have the same meaning as in Fig. 4.

lent flows, where the formation of density enhancements is expected to take place (see Sect. 5.9). The timescale for the dissipation of turbulence in dense cores is comparable to the freefall timescale (e.g., Mac Low \& Klessen 2004). Thus, because the observed linewidth-size relation appears to be quite flat, it is possible that the turbulent gas motions at small scales $(\lessgtr 0.1 \mathrm{pc})$ are not yet settled into equilibrium state (i.e., relaxed). In this case, the core formation should be a rapid process with the corresponding timescale being comparable to the free-fall time.

\subsection{Internal pressure support}

The internal kinetic gas pressure, $P_{\text {int }}$, within the core consists of a thermal pressure, $p_{\mathrm{T}}=n k_{\mathrm{B}} T_{\mathrm{kin}}$, and a non-thermal pressure, $p_{\mathrm{NT}}=\mu m_{\mathrm{H}} n \sigma_{\mathrm{NT}}^{2}$, where $n$ is the number density, and $\mu=2.33$ $\left(P_{\text {int }}=p_{\mathrm{T}}+p_{\mathrm{NT}}\right)$. In the left panel of Fig. 7 , we plot $P_{\text {int }} / k_{\mathrm{B}}$ versus core mass. Excluding the one outlier in the plot (SMM 6), there is a trend of increasing internal pressure with increasing core mass. This indicates that the cores are not in pressure equilibrium with an external pressure due to, e.g., the weight of the cloud in which the cores are embedded (Lada et al. 2008). The mean internal pressure of the Orion B9 core population is $\sim 1.1 \times 10^{6} \mathrm{~K} \mathrm{~cm}^{-3}$. This is in excellent agreement with the value $\sim 10^{6} \mathrm{~K} \mathrm{~cm}^{-3}$ estimated by Johnstone et al. (2001) for the cores in the northern part of Orion B. For comparison, the overall pressure of the ISM in the Galactic midplane, which consists of thermal and turbulent kinetic pressures, and the pressures of magnetic fields and cosmic rays, has been estimated to be $P_{\mathrm{ISM}} / k_{\mathrm{B}} \approx 2.8 \times 10^{4} \mathrm{~K} \mathrm{~cm}^{-3}$ (Boulares \& Cox 1990). The high pressures inside cores are likely to result from compression by gravity (see Sect. 5.4).

To examine the relative role of turbulence in the core internal pressure, we calculated the ratio of thermal to non-thermal pressure, which is given by $R_{\mathrm{p}}=1 / f_{\text {turb }}^{2}$ (see Sect. 4.1.4). The values of $R_{\mathrm{p}}$ are plotted as a function of mass in the right panel of Fig. 7. As can be seen in this figure, thermal pressure is clearly the dominant source of internal gas pressure for 8 of 11 cores. For the rest of the cores, thermal pressure support is still significant as they have $R_{\mathrm{p}}>0.5$. Also in this regard, the Orion B9 cores appear to be similar to low-mass dense cores in nearby molecular clouds, which are commonly found to be thermally dominated (e.g., Myers \& Benson 1983; Kirk et al. 2007; Lada et al. 2008). 

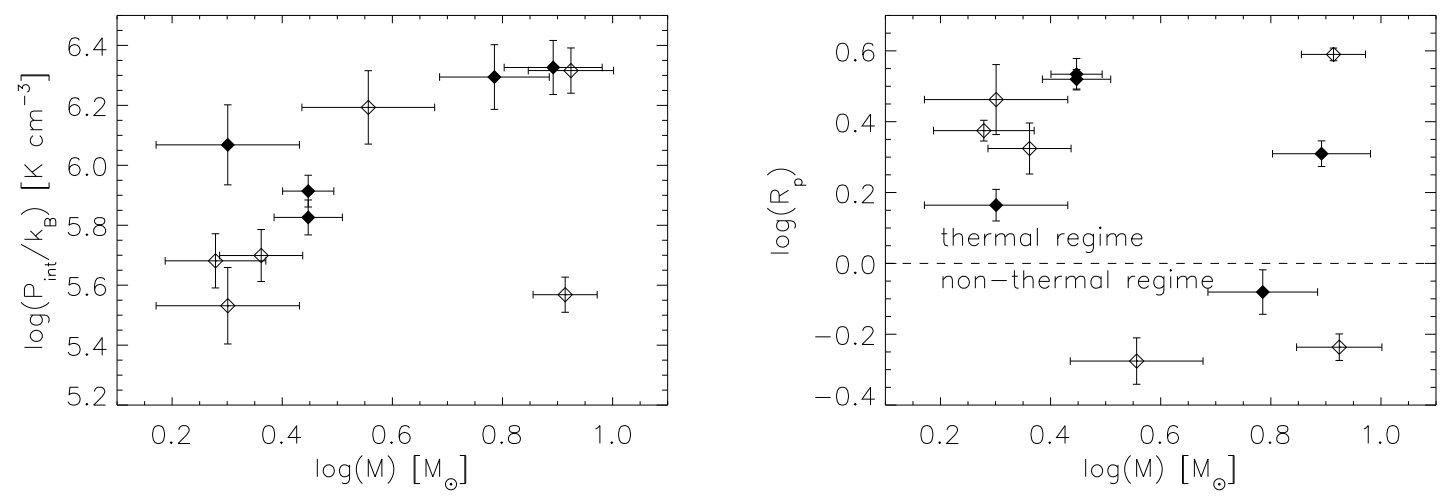

Fig. 7. Left: total internal kinetic gas pressure versus core mass. Right: the ratio of thermal to non-thermal pressure as a function of mass. The dashed horizontal line marks the point at which thermal and non-thermal pressure support are equal. Both plots are in log-log scales, and the symbols have the same meaning as in Fig. 4.

\subsection{Dynamical state and gravitational boundedness of the cores}

To further examine the dynamical state of the cores, we inspect the virial parameters derived in Sect. 4.4. Figure 8 (left panel) shows the distribution of the virial parameter for the Orion B9 cores as a function of mass. Within the errors, all eleven cores are self-gravitating $\left(\alpha_{\mathrm{vir}}<2\right)$, and five of them seem to be close to the virial equilibrium or collapsing $\left(\alpha_{\text {vir }} \leq 1\right)$. One should note that the virial calculation presented above did not include any external pressure or magnetic fields. An external presssure of $P_{\text {ext }} / k_{\mathrm{B}} \sim 2-3 \times 10^{5} \mathrm{~K} \mathrm{~cm}^{-3}$ would bring all starless cores except SMM 7 to virial equilibrium or make them collapse (neglecting the possible magnetic support). Assuming that the gas surrounding the cores is characterised by $\sigma_{\mathrm{NT}} \sim 0.6 \mathrm{~km} \mathrm{~s}^{-1}$ and $n\left(\mathrm{H}_{2}\right)=2 \times 10^{3} \mathrm{~cm}^{-3}$, corresponding to the typical width of ${ }^{13} \mathrm{CO}(1-0)$ lines observed by Caselli \& Myers (1995) in this region and the critical density of the transition in question (which is lower than the critical density of the $\mathrm{NH}_{3}$ inversion lines), its turbulent ram pressure would roughly equal to the required confining pressure quoted above. As discussed by Lada et al. (2008) in the case of Pipe Nebula, the supersonic intercore turbulence can be a manifestation of the self-gravity of the surrounding massive cloud.

It seems likely that most if not all starless cores in Orion B9 detected in this survey are prestellar, i.e. they will eventually collapse to stars. The situation resembles that observed in Perseus by Foster et al. (2009): most cores are gravitationally bound, and $\sim 1 / 3$ are in virial equilibrium.

The virial parameter, $\alpha_{\text {vir }}$ seems to decrease as a function of core mass (see Fig. 8, left). The slope of a least-squares fit, $\log \left(\alpha_{\text {vir }}\right)=(0.54 \pm 0.10)-(0.67 \pm 0.16) \log (M)$, with the linear correlation coefficient $r=-0.81$, is very close to that found by Lada et al. (2008) for cores in the Pipe Nebula which are believed to be predominantly confined by external pressure. Our cores with masses in the range $2-8 M_{\odot}$ correspond to the most massive Pipe cores. The slope $(\sim-2 / 3)$ is consistent with the theoretical prediction of Bertoldi \& McKee (1992) for pressure confined cores, and basically results from the fact that there is not much core-to-core variation in the average densities and velocity dispersions. It should be noted, however, that the overall level of $\alpha_{\mathrm{vir}}$, characterised by the constant of the fit, is lower than derived in the Pipe, probably reflecting the different environments and evolutionary stages of the two regions.

Like Lada et al. (2008) we have also plotted in the right panel of Fig. 8 the correlation diagram between the ratio $\sigma_{3 \mathrm{D}} / v_{\mathrm{esc}}$ and the core mass, where the three-dimensional velocity dispersion, $\sigma_{3 \mathrm{D}}$, is calculated from $\sigma_{3 \mathrm{D}}=\sqrt{3 c_{\mathrm{s}}^{2}+3 \sigma_{\mathrm{NT}}^{2}}$, and the escape velocity, $v_{\text {esc }}$ is given by $v_{\text {esc }}=\sqrt{2 G M / R_{\text {eff }}}$. For all cores, $\sigma_{3 \mathrm{D}}$ is smaller than $v_{\mathrm{esc}}$, supporting the above conclusion that the cores are gravitationally bound. Morever, the $\sigma_{3 \mathrm{D}} / v_{\mathrm{esc}}$ ratio appears to decrease as a function of core mass. A least-squares fit to the whole sample gives $\log \left(\sigma_{3 \mathrm{D}} / v_{\mathrm{esc}}\right)=(-0.01 \pm 0.03)-$ $(0.43 \pm 0.05) \log (M)$, with $r=-0.94$. This relationship implies that the transition from unbound to bound core occurs at about $0.8-0.9 M_{\odot}$. This is very close to the peak of the CMF for Orion B North, i.e., $\sim 1 M_{\odot}$ (Nutter \& Ward-Thompson 2007). This is expected because the CMFs for Orion B9 and Orion B North seem to represent the subsamples of the same parent distribution (Sect. 4.3). A similar correspondence was found by Lada et al. (2008) for the dense cores in the Pipe Nebula.

\subsection{Correlating the properties of protostellar cores}

In Paper I, we derived the SEDs for the protostellar cores in Orion B9. For all sources, the observed flux densities were fitted by a two-temperature (warm+cold) composite model. The bolometric dust temperatures and bolometric luminosities of the sources derived from the SEDs are given in Table 6 of Paper I.

In the left panel of Fig. 9, we show the correlation plot between $T_{\text {kin }}$ and the bolometric temperature, $T_{\text {bol }}$. Only for SMM 3, the two values are similar within the errors, and in the case of SMM $4, T_{\text {kin }}$ and $T_{\text {bol }}$ agree within a few kelvins. For the other sources (i.e., the three IRAS sources), however, the temperatures estimated from the SEDs are clearly higher than those obtained from $\mathrm{NH}_{3}$ measurements. The SEDs of SMM 3 and 4 were constructed by using only three flux density values at 24, 70, and $870 \mu \mathrm{m}$ (see Paper I; Fig. 6 therein). In the case of other sources, also the flux densities at all four IRAS bands (12, 25,60 , and $100 \mu \mathrm{m}$ ) were included in the SED fits. This may have caused the temperature of the cold part of the spectrum to be overestimated. Shetty et al. (2009) recently concluded that if using flux density values at widely separated wavelengths, and including short-wavelength flux densities in the SED fit, the obtained temperatures may be too high.

The right panel of Fig. 9 shows a plot of $\Delta v_{\mathrm{NT}}$ as a function of $L_{\text {bol }}$. There appears to be a positive correlation between the two quantities, and the least-squares linear fit to the data gives $\log \left(\Delta v_{\mathrm{NT}}\right)=(-0.65 \pm 0.10)+(0.25 \pm 0.11) \log \left(L_{\mathrm{bol}}\right)$, with $r=$ 0.80 . We also show the relationships found by Jijina et al. (1999) 

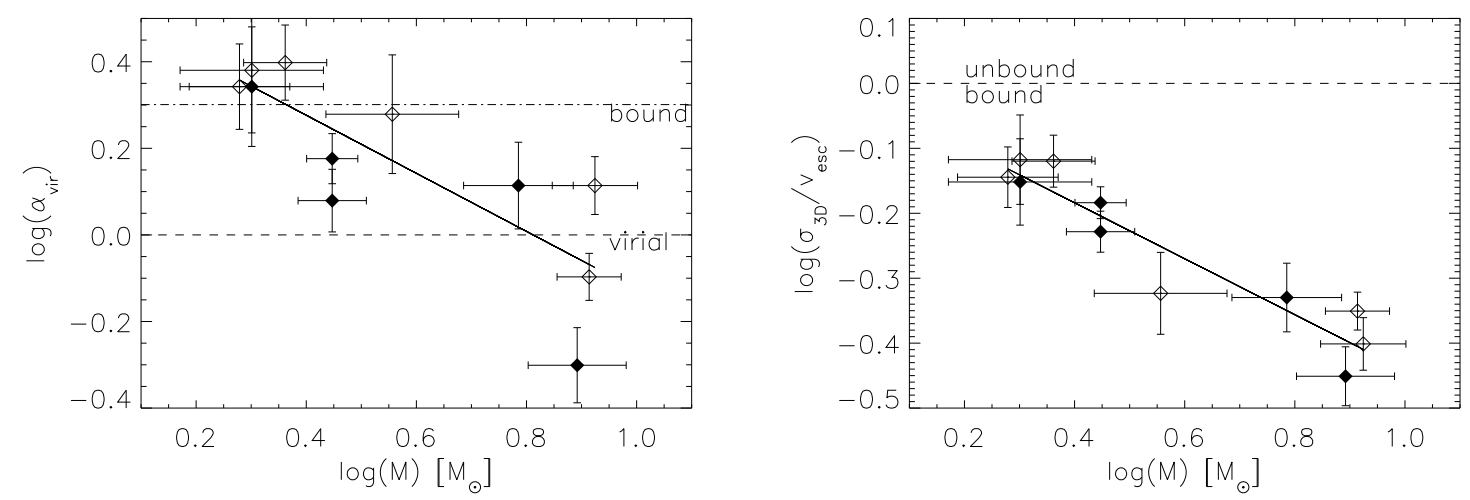

Fig. 8. Left: virial parameter versus core mass. The dashed line indicates the virial equilibrium limit $\left(\alpha_{\text {vir }}=1\right)$, and the dash-dotted line shows the limit of gravitational boundedness $\left(\alpha_{\text {vir }}=2\right)$. Right: ratio of 3D velocity dispersion to escape velocity against core mass. The dashed line indicates $\sigma_{3 \mathrm{D}} / v_{\mathrm{esc}}=1$. All cores are likely to be gravitationally bound. The solid line in both plots indicates the least-squares fit to the data. Both plots are in log-log scales, and the symbol key is identical to that of Fig. 4.
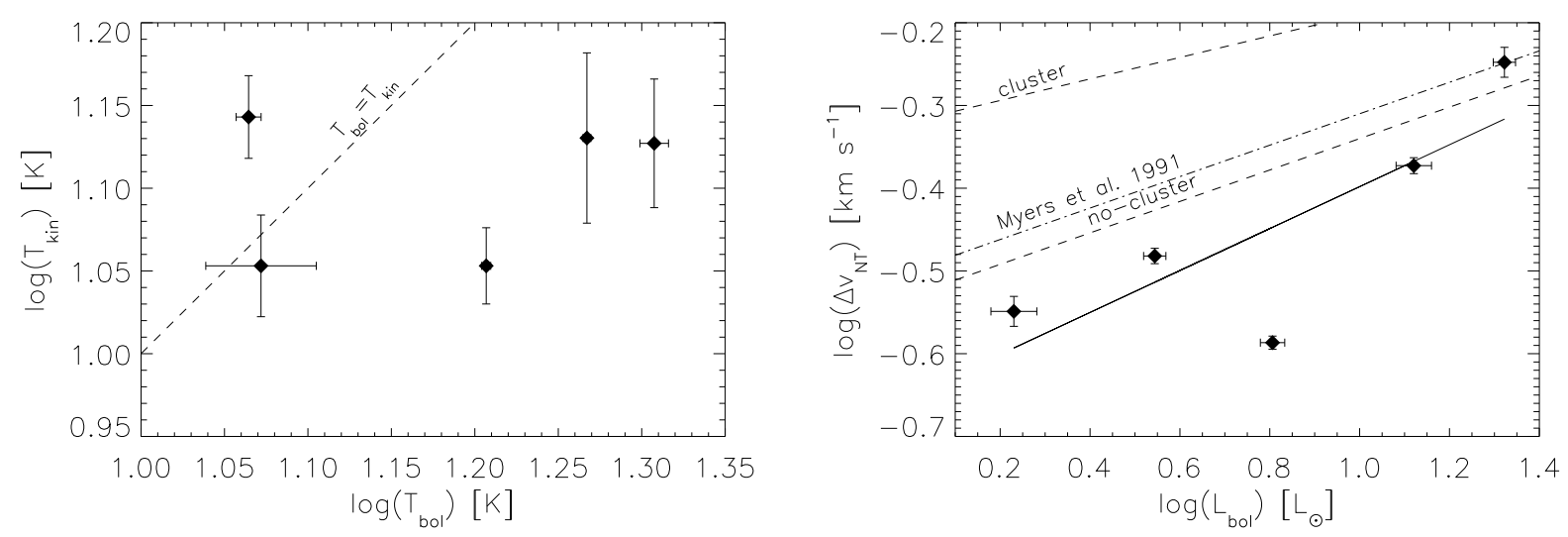

Fig. 9. Left: $T_{\text {kin }}$ versus $T_{\text {bol }}$. The dashed line indicates $T_{\text {kin }}=T_{\text {bol }}$. Right: $\Delta v_{\mathrm{NT}}$ versus $L_{\mathrm{bol}}$. The solid line indicates the least-squares fit to the data. The upper and lower dashed lines represent relationships found by Jijina et al. (1999) for $\mathrm{NH}_{3}$ cores with and without cluster association, respectively. The dash-dotted line shows the relationships found by Myers et al. (1991) for IRAS point sources. Both plots are in log-log scales.

for $\mathrm{NH}_{3}$ cores in clusters and cores without cluster association (see their Table B7), and the Myers et al. (1991) relation for a sample of 61 IRAS point sources (their Eq. (3)). Our result is similar to the relationship found in the latter two studies, $\Delta v_{\mathrm{NT}} \propto L_{\mathrm{bol}}^{0.19}$. The fact that $\Delta v_{\mathrm{NT}}$ increases as a function of $L_{\mathrm{bol}}$ can be explained by the effects of winds driven by the embedded protostar+disk system, and by the fact that more turbulent initial conditions give rise to more massive, i.e., more luminous stars (Myers et al. 1991). As in the case of $\Delta v_{\mathrm{NT}}-\Delta v_{\mathrm{T}}$ relation (see Fig. 5), the Orion B9 cores more closely follow the relationship for isolated cores.

\subsection{Column densities and fractional abundances of $\mathrm{NH}_{3}$ and $\mathrm{N}_{2} \mathrm{H}^{+}$}

The mean $\mathrm{NH}_{3}$ fractional abundance in our sample is $4.8 \pm 0.7 \times$ $10^{-8}$, where the \pm -error represents the standard deviation of the mean. The value of $\left\langle x\left(\mathrm{NH}_{3}\right)\right\rangle$ in Orion $\mathrm{B} 9$ is similar to those recently found by Foster et al. (2009) and Friesen et al. (2009) for the dense cores in Perseus and Ophiuchus, respectively (i.e., a few to several $\times 10^{-8}$ ). For comparison, the $\mathrm{NH}_{3}$ abundances in dense cores in nearby regions of isolated star formation, such as Taurus, are commonly found to be $\sim 0.4-5 \times 10^{-8}$ (e.g., Hotzel et al. 2001; Tafalla et al. 2002, 2006). The average $\mathrm{N}_{2} \mathrm{H}^{+}$ abundance in Orion $\mathrm{B} 9,\left\langle x\left(\mathrm{~N}_{2} \mathrm{H}^{+}\right)\right\rangle=2.5 \pm 0.8 \times 10^{-10}$, is very similar to those in isolated low-mass cores (e.g., $\left\langle x\left(\mathrm{~N}_{2} \mathrm{H}^{+}\right)\right\rangle=$ $2 \pm 1 \times 10^{-10}$ and $3 \pm 2 \times 10^{-10}$ for the starless and protostellar core samples of Caselli et al. (2002)). On the other hand, the value of $\left\langle x\left(\mathrm{~N}_{2} \mathrm{H}^{+}\right)\right\rangle$is about $2-6$ times lower than those determined by Friesen et al. (2010) in the Oph B1 and B2 cores which lie in the region of clustered star formation.

As shown in the left panel of Fig. 10, the fractional $\mathrm{NH}_{3}$ abundance appears to decrease with increasing $\mathrm{H}_{2}$ column density (as traced by $870 \mu \mathrm{m}$ continuum emission smoothed to $40^{\prime \prime}$ resolution). A linear-least-squares fit to the data gives $\log x\left(\mathrm{NH}_{3}\right)=(4.2 \pm 6.6)-(0.5 \pm 0.3) \log N\left(\mathrm{H}_{2}\right)$, with $r=-0.51$. The slope of this relationship, $x\left(\mathrm{NH}_{3}\right) \propto 1 / \sqrt{N\left(\mathrm{H}_{2}\right)}$, is very similar to that found by Friesen et al. $(2009,2010)$ in the Oph B2 core. In the middle panel of Fig. 10, we plot $x\left(\mathrm{NH}_{3}\right)$ as a function of $n\left(\mathrm{H}_{2}\right)$. The trend is similar as above, and the least-squares linear fit to the data indicates $\log x\left(\mathrm{NH}_{3}\right)=(-4.5 \pm 1.4)-(0.6 \pm$ 0.3) $\log n\left(\mathrm{H}_{2}\right)$, with $r=-0.57$. The tendencies described above are likely to manifest the fact that $\mathrm{NH}_{3}$, like species containing carbon or oxygen, accrete onto grain surfaces at high densities. According to chemistry models of Aikawa et al. (2005) and Flower et al. (2006), the $\mathrm{NH}_{3}$ abundance becomes heavily depleted at densities $n_{\mathrm{H}} \gtrsim$ a few $\times 10^{6} \mathrm{~cm}^{-3}$ (due to freeze-out of the parent species $\left.\mathrm{N}_{2}\right)$. The $x\left(\mathrm{NH}_{3}\right)-n\left(\mathrm{H}_{2}\right)$ relationship shown in Fig. 10 agrees reasonably well with the predictions for the early stages of collapse presented in Flower et al. (2006; see, e.g., their Fig. 4). In the Flower et al. model, the protostellar 

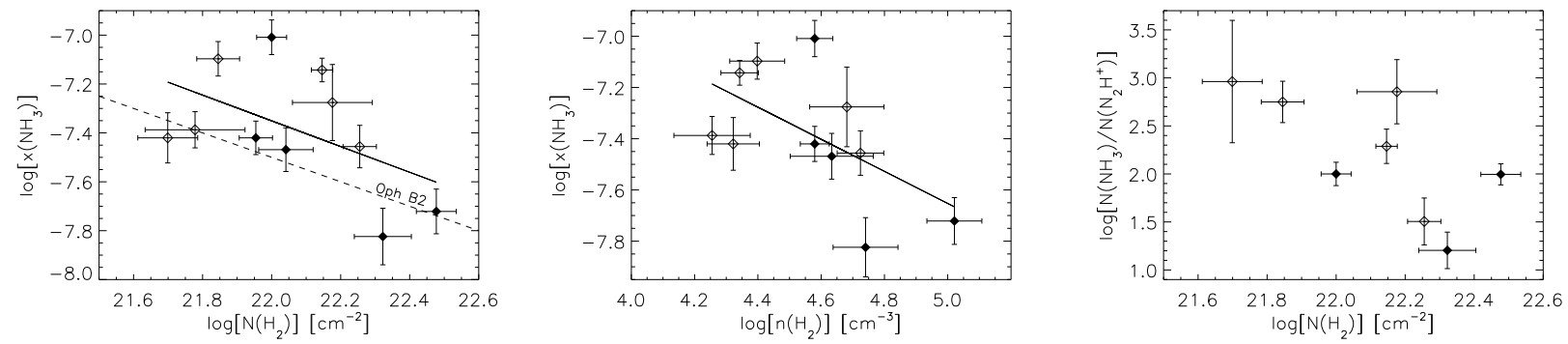

Fig. 10. Comparison of chemical abundances as a function of $\mathrm{H}_{2}$ column and number density. All plots are in log-log scales, and the symbol key is identical to that of Fig. 4. The solid lines indicate the least-squares fits to the data. Left: $x\left(\mathrm{NH}_{3}\right)$ versus $N\left(\mathrm{H}_{2}\right)$. The dashed line indicates the relationship found by Friesen et al. $(2009,2010)$ for Oph B2. Middle: $x\left(\mathrm{NH}_{3}\right)$ versus $n\left(\mathrm{H}_{2}\right)$. Right: $\mathrm{NH}_{3} / \mathrm{N}_{2} \mathrm{H}^{+}$column density ratio versus $N\left(\mathrm{H}_{2}\right)$.

collapse occurs on the free-fall timescale which is about $4 \times$ $10^{5} \mathrm{yr}$ at $n_{\mathrm{H}}=10^{4} \mathrm{~cm}^{-3}$. This is in agreement with statistical estimates of the prestellar core lifetime (see Sect. 5.9).

Unlike in Friesen et al. (2010), no correlation was found between $x\left(\mathrm{~N}_{2} \mathrm{H}^{+}\right)$and $\mathrm{H}_{2}$ column or number densities. In the right panel of Fig. 10, we plot the $\mathrm{NH}_{3} / \mathrm{N}_{2} \mathrm{H}^{+}$column density ratio as a function of $N\left(\mathrm{H}_{2}\right)$ (determined using $40^{\prime \prime}$ resolution). A possible negative correlation can be seen between the two quantities, resembling the result found by Friesen et al. (2010) in Oph B1, and by Johnstone et al. (2010) in Perseus. As can be seen from the figure and Col. (4) of Table 5, prestellar cores generally have higher values of $N\left(\mathrm{NH}_{3}\right) / N\left(\mathrm{~N}_{2} \mathrm{H}^{+}\right)$ratio than in protostellar cores. The tendency has been observed also in other starforming regions (Paper I and references therein; Friesen et al. 2010). The reason for this differentiation is not quite clear. The abundances of both molecules build up slowly, and they benefit from increasing density and the freezing of $\mathrm{CO}$ and other heavier species (e.g., Aikawa et al. 2005). For both molecules the enhanced formation at high densities should be counteracted by accretion onto dust grains. In the time evolution of the column densities these contradictory tendencies are probably reflected as $\mathrm{NH}_{3}$ and $\mathrm{N}_{2} \mathrm{H}^{+}$peaks coming one after the other. Because of the different radial distributions of these molecules (and probably also because of the different critical densities of their transitions) the derived column density ratios depend on the spatial resolution. The fact that Friesen et al. (2010) could see the effect of $\mathrm{N}_{2} \mathrm{H}^{+}$depletion towards the highest densities can probably be explained by a higher resolution and better data quality than available here.

Our data contains, however, evidence that $\mathrm{N}_{2} \mathrm{H}^{+}$is frozen out in the core SMM 4, as suggested in Paper I. The core was not detected in $\mathrm{N}_{2} \mathrm{H}^{+}(3-2)$, and the absence of this molecule is most probably not caused by efficient desorption of $\mathrm{CO}$, which is one of the principal destructors of $\mathrm{N}_{2} \mathrm{H}^{+}$in the gas phase. The gas kinetic temperature derived from ammonia $(\sim 14 \mathrm{~K})$ is clearly lower than the sublimation temperature of $\mathrm{CO}$ ( $20 \mathrm{~K}$; Aikawa et al. 2008). Depletion of $\mathrm{N}_{2} \mathrm{H}^{+}$could also be the reason for the very weak $\mathrm{N}_{2} \mathrm{H}^{+}(3-2)$ emission seen towards Ori B9 N. An other indication of depletion in the core Ori B9 $\mathrm{N}$ is that the $\mathrm{H}_{2} \mathrm{D}^{+}\left(1_{10}-1_{11}\right)$ line at $\sim 9 \mathrm{~km} \mathrm{~s}^{-1}$ is detected in this source, about $38^{\prime \prime}$ southeast from the dust peak position (Harju et al. 2006). This molecule is known to resist depletion "to last" and is observed in highly depleted cold cloud cores (Caselli et al. 2008).

\subsection{Low-velocity gas emission seen towards Orion B9}

The LSR velocities of SMM $7\left(3.6 \mathrm{~km} \mathrm{~s}^{-1}\right)$ and IRAS 05413$0104\left(1.5 \mathrm{~km} \mathrm{~s}^{-1}\right)$ are about $6-8 \mathrm{~km} \mathrm{~s}^{-1}$ less than the systemic velocity of Orion B (see Sect. 3.1). We note that the systemic velocity of IRAS 05413-0104 is known to be low before the present study (e.g., from $\mathrm{NH}_{3}$ measurements by Wiseman et al. 2001). Given that the sources north-east from the central part of Orion B9 appear to have lower radial velocities, the same is likely to be true for IRAS 05412-0105. Indeed, IRAS 054120105 and 05413-0104 could be embedded in a common $1.2 \mathrm{~mm}$ clump (Kauffmann et al. 2008). $\mathrm{No} \mathrm{NH}_{3}(1,1)$ line was detected towards IRAS 05412-0105 (Sect. 2.1), and we could not found any other spectral line observations for it from the literature (providing kinematic information). Also, the second velocity components seen in the $\mathrm{NH}_{3}$ and $\mathrm{N}_{2} \mathrm{H}^{+}(3-2)$ spectra towards $\mathrm{SMM}$ 4 and Ori B9 $\mathrm{N}$ have centroid velocities $\sim 7-8 \mathrm{~km} \mathrm{~s}^{-1}$ lower than the systemic velocity. In Paper I, the $\sim 2 \mathrm{~km} \mathrm{~s}^{-1}$ velocity components were detected in $\mathrm{N}_{2} \mathrm{H}^{+}(1-0)$ and/or $\mathrm{N}_{2} \mathrm{D}^{+}(2-1)$ towards a few target positions near IRAS 05405-0117 (called Ori B9 E in Paper I) and Ori B9 N. Similarly, Aoyama et al. (2001) found that the $\mathrm{C}^{18} \mathrm{O}(1-0)$ and $\mathrm{H}^{13} \mathrm{CO}^{+}(1-0)$ clumps associated with IRAS 05405-0117 have centroid LSR velocities of about $2 \mathrm{~km} \mathrm{~s}^{-1}$ (at the resolutions 2.7 and 3.8, respectively). This raises the question whether the sources and/or high-density gas showing the lower velocity emission are associated with the same cloud complex as the "regular" $9 \mathrm{~km} \mathrm{~s}^{-1}$ component?

The $\mathrm{CO}(1-0)$ integrated intensity maps in the velocity ranges $-1.0-2.3$ and $2.3-5.5 \mathrm{~km} \mathrm{~s}^{-1}$ by Wilson et al. (2005, their Fig. 3) show relatively strong emission in the direction of Orion B9. As discussed by Wilson et al. (2005), the stellar winds from the Ori OB $1 \mathrm{~b}$ subgroup have likely interacted with the lowlongitude and -latitude part of Orion B, resulting in an accelerated motion towards the Sun. This fraction of the gas is likely to be located a few tens of parsecs closer to the Sun than the higher velocity gas, and it is estimated to have a mass of about $15 \%$ of the total mass of Orion B, i.e., $\sim 10^{4} M_{\odot}$ (Wilson et al. 2005). Our pointed molecular line observations in high density tracers, including deuterated species, show that the low-velocity gas in Orion B also contains dense cores, possibly originating from the fragmentation of the cloud region compressed by the feedback from the massive stars of the Ori OB 1 group.

\subsection{Core spatial distribution revisited}

In Paper I, we studied the spatial distribution of cores in Orion B9 in order to examine the fragmentation length-scale. We determined the core-separation distribution and the number distribution of the projected separation distance between nearest neighbours. These were compared with the corresponding random distributions. We concluded that the observed distributions are random-like, and thus the origin of cores is possibly caused by turbulent fragmentation; random distribution is 
expected if the cloud fragmentation is driven by a stochastic turbulent process. As discussed in the previous subsection, some of the cores are probably located at somewhat different distance than the "9 $\mathrm{km} \mathrm{s}^{-1}$ members of Orion B9". For this reason, we re-examined the core spatial distribution in the region.

We note furthermore that there was a flaw in our IDL procedure used to generate the histograms shown in Fig. 8 of Paper I. The number of separations (66 for the core separations, and 12 for the nearest neighbours) were not correct, and the numbers in the observed and model (random) distributions were not equal. Here we reproduce the histograms and show the new plots for the core separations and nearest neighbours in the top panels of Fig. 11. In the bottom panels of Fig. 11, we show the same distributions but without the sources SMM 7, IRAS 05412-0105, and IRAS 05413-0104. In the latter figure, the size of the region used to generate the random distributions was decreased from $0.22 \square^{\circ}$ to $0.06 \square^{\circ}$. The obtained statistics of the distributions are presented in Table 7 . In this table, we give the mean (and its standard deviation) and median of the observed core spatial distribution (Cols. (2) and (3)), those of the corresponding random distribution (Cols. (4) and (5)), ratios between the observed and random mean and median separations (Cols. (6) and (7)), and probability given by the two-sample K-S test that the observed and random distributions are drawn from the same underlying distribution, see below (Col. (8)). The random distributions were generated a hundred times and the averaged histograms were compared with those derived from observations. The numerical values for the random distributions presented in Table 7 are the averages and the standard deviations of these 100 runs. Only a few values differ from those reported in Paper I (see the footnotes in the table). Also, for the core positions in Orion B North (Nutter \& Ward-Thompson 2007), the values are the same as those reported in Paper I. We note that for the core-separation distributions $\langle r\rangle_{\text {OriB9 }} /\langle r\rangle_{\text {OriBN }}=0.63 \pm 0.01$ and $\tilde{r}_{\text {OriB9 }} / \tilde{r}_{\text {OriBN }}=0.62$, and for the nearest-neighbour distribution $\langle r\rangle_{\text {OriB9 }} /\langle r\rangle_{\text {OriBN }}=1.95 \pm 0.27$ and $\tilde{r}_{\text {OriB9 }} / \tilde{r}_{\text {OriBN }}=1.87$.

In Paper I, we suggested that the comparable mean and median values between the observed and random distributions indicate that the cores are likely to be randomly distributed within the region. To examine this in more detail, we carried out K-S tests between the observed and model distributions. As shown in the last column of Table 7, the probability that the observed distribution and the generated random distribution represent the same underlying distribution is very small in all cases. Such low probabilities call into question the similarity between the observed and random distributions. Thus, even the observed and model mean and median separation-distances are comparable, the above K-S test probabilities suggest that the distributions as a whole are not similar. The K-S probability is expected to be a more robust measure of the similarity between the two distributions than the comparison of the mean and median values because the latter two can be the same for two different distributions.

The observed mean and median distances between the nearest-neighbours are in the range $\sim 3 \times 10^{4}-6 \times 10^{4}$ AU. Assuming that the parental cloud region is characterised by the average gas kinetic temperature and density values of $15 \mathrm{~K}$ and $\sim 2 \times 10^{3}-10^{4} \mathrm{~cm}^{-3}$, respectively (Sect. 5.4), the thermal Jeans length, $\lambda_{\mathrm{J}}=\sqrt{\pi c_{\mathrm{s}}^{2} / G \rho}$, is about $5 \times 10^{4}-10^{5} \mathrm{AU}$. These are comparable to the observed core separations indicated above. This suggests that the fragmentation of the region into cores is caused by gravitational instability. The parental cloud region in which the cores have formed could have been initially compressed by the winds from the nearby massive stars as discussed in Sect. 5.7.

\subsection{The lifetime and origin of dense cores in Orion B9}

According to the scenario of turbulence-regulated star formation, the prestellar core evolution is dynamic and the corresponding lifetime is only a few times the free-fall time (Mac Low \& Klessen 2004; Vázquez-Semadeni et al. 2005). Several results of our study conform to this scenario: as was discussed in Sect. 5.2, the Orion B9 cores could have been formed in turbulent shocks where the dissipation of the kinetic energy of turbulent motions took place. It was also concluded that the timescale for core formation should be comparable to the free-fall time. On the other hand, the abundances of the $\mathrm{N}$-bearing species studied in the present paper are consistent with those predicted by chemical models based on dynamical prestellar core evolution (Sect. 5.6). Moreover, in Paper I we deduced that there are equal numbers of pre- and protostellar cores in the Orion B9 region, which suggest that the corresponding lifetimes are comparable. Based on similar statistical results, i.e., $N_{\text {pre }} / N_{\text {proto }} \approx 1$, recent studies have shown that the duration of the prestellar phase of core evolution at densities $n\left(\mathrm{H}_{2}\right) \gtrsim 10^{4} \mathrm{~cm}^{-3}$ is only a few times the free-fall time (see references in Paper I; Evans et al. 2009). It is possible that some of the cores do not belong to the same volume of the cloud as those with centroid velocities around $9 \mathrm{~km} \mathrm{~s}^{-1}$. Even if the cores with lower centroid velocities are excluded (i.e., SMM 7, and IRAS 05413-0104 and 05412-0105), the above ratio is $5: 4=1.25$, which is still consistent with the dynamic core evolution scenario.

In the present study (and in Paper I), we found that the CMFs in Orion B9 and Orion B North very likely represent the subsamples of the same parent distribution, and resemble the stellar IMF (Nutter \& Ward-Thompson 2007). Numerical simulations of turbulent fragmentation have been able to reproduce the general shape of the IMF (e.g., Padoan \& Nordlund 2002; Ballesteros-Paredes et al. 2006). On the other hand, recent models concerning fragmentation initiated by the ambipolar diffusion (AD) have likewise succeeded in explaining the connection between the CMF and the IMF (Kunz \& Mouschovias 2009).

In Paper I, we used the spatial distribution of cores, which seemed to mimic a random distribution, as an argument for the dominance of turbulent fragmentation in core formation. Moreover, we stated that because region represents clustered star formation, the turbulence is most likely driven on large scales (e.g., Klessen et al. 2000; Klessen 2001). However, the present molecular line data and a more careful analysis of the spatial distribution have called both arguments into question: According to the K-S test, the core distribution is unlikely to be random, and the core properties resemble closely those in the regions of isolated star formation.

To summarise, the current data suggest that the evolution of the Orion B9 cores is dynamic. As discussed in Sect. 5.7, the Orion B9 region is likely to be influenced by the massive stars of the Ori OB $1 \mathrm{~b}$ group. This interaction process could have led to the driving of the large-scale turbulence and/or to the compression of the cloud material into dense sheets and filaments as observed in molecular clouds. These structures could have then been fragmented into dense cores via gravitational instability as suggested by the Jeans-length analysis in Sect. 5.8. Further investigations of the chemistry, deuteration, and the degree of ionisation can help to fix the evolutionary timescales, and thereby estimate the roles of turbulence, 

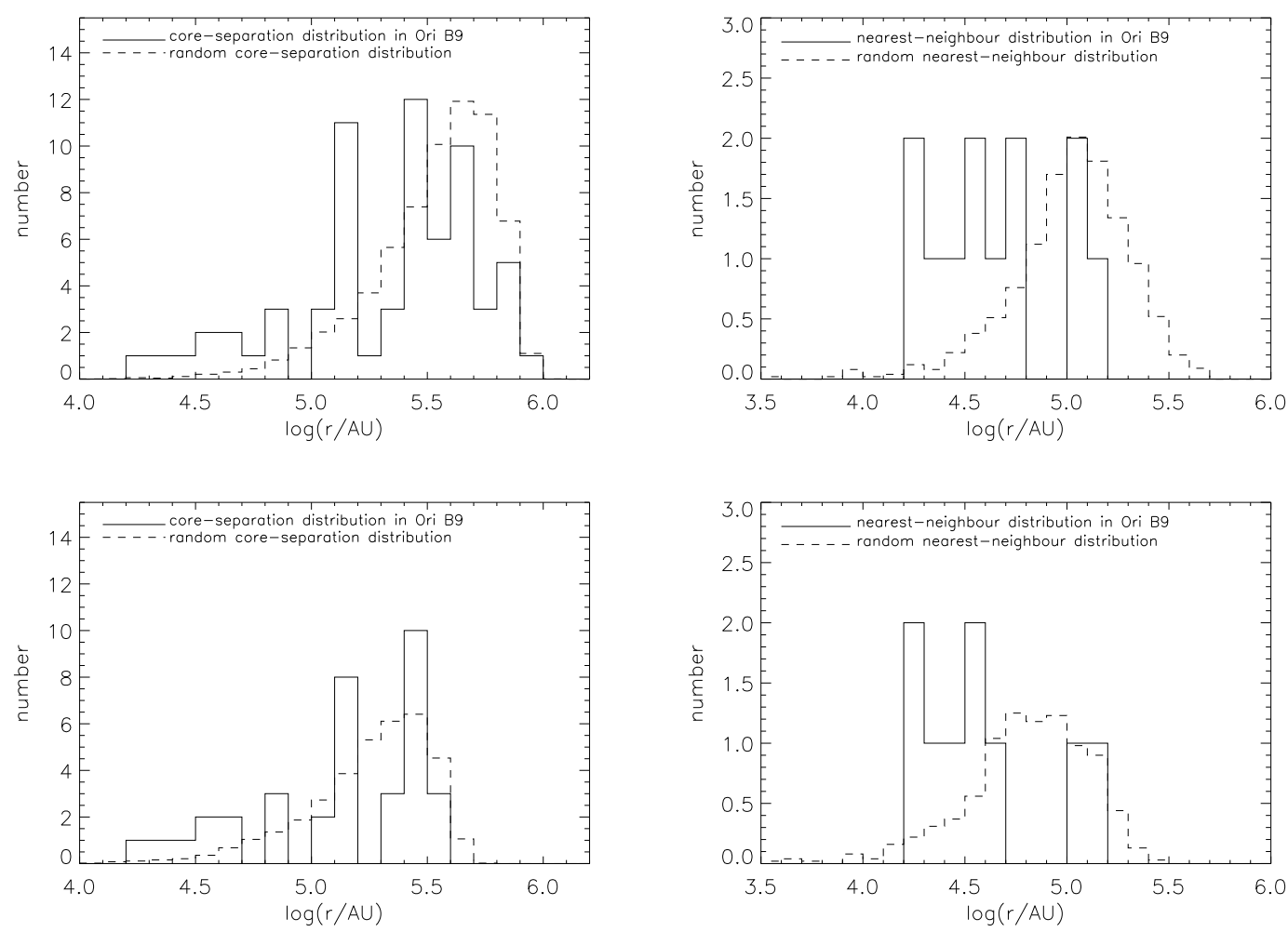

Fig. 11. Top left: observed core-separation distribution (solid line) compared with the expected distribution for random distribution of the same number of cores as the observed sample over an identical area (dashed line). Top right: same as in the top left panel but for the nearest-neighbour distribution. Bottom panels: same as in the top panels, but SMM 7, IRAS 05412-0105, and IRAS 05413-0104 have been excluded from the samples.

Table 7. Statistics of the core spatial distributions in Orion B9.

\begin{tabular}{|c|c|c|c|c|c|c|c|}
\hline & $\begin{array}{c}\langle r\rangle_{\mathrm{obs}} \\
{[\log \mathrm{AU}]}\end{array}$ & $\begin{array}{c}\tilde{r}_{\text {obs }} \\
{[\log \mathrm{AU}]}\end{array}$ & $\begin{array}{c}\langle r\rangle_{\mathrm{ran}} \\
{[\log \mathrm{AU}]}\end{array}$ & $\begin{array}{c}\tilde{r}_{\text {ran }} \\
{[\log \mathrm{AU}]}\end{array}$ & $\langle r\rangle_{\mathrm{obs}} /\langle r\rangle_{\mathrm{ran}}$ & $\tilde{r}_{\mathrm{obs}} / \tilde{r}_{\mathrm{ran}}$ & $\begin{array}{c}\text { Prob. } \\
{[\%]}\end{array}$ \\
\hline \multicolumn{8}{|c|}{ Core separation-distribution } \\
\hline Original sample & $5.467=$ & 5.420 & $5.525 \pm 0.051^{a}$ & $5.584 \pm$ & 0.8 & 0.69 & $1.7 \pm 0.6$ \\
\hline Reduced sample ${ }^{b}$ & $5.246=$ & 5.195 & $5.245 \pm 0.068$ & 5.305 & $1 .($ & 0.7 & 4.7 \\
\hline \multicolumn{8}{|c|}{ Nearest neighbour-distribution } \\
\hline Original sample & $4.750 \pm 0.090$ & 4.619 & $5.018 \pm 0.047^{c}$ & $5.035 \pm 0.045^{d}$ & $0.54 \pm 0.05$ & $0.38 \pm 0.04$ & $5.7 \pm 1.2$ \\
\hline Reduced sample $^{b}$ & $4.699 \pm 0.123$ & 4.511 & $4.806 \pm 0.130$ & $4.822 \pm 0.144$ & $0.78 \pm 0.01$ & $0.49 \pm 0.16$ & $23.8 \pm 2.6$ \\
\hline
\end{tabular}

Notes. ${ }^{(a)}$ In Paper I, this was reported to be $5.59 \pm 0.05 .{ }^{(b)}$ SMM 7, IRAS 05412-0105, and IRAS 05413-0104 have been excluded from the sample. ${ }^{(c)} 5.08 \pm 0.08$ in Paper I. ${ }^{(d)} 5.07 \pm 0.11$ in Paper I.

thermo-gravitational instability, and ambipolar diffusion in the core formation.

\section{Summary and conclusions}

We observed the $\mathrm{NH}_{3}(1,1)$ and $(2,2)$ inversion transitions, and the $\mathrm{N}_{2} \mathrm{H}^{+}(3-2)$ lines towards the submm cores in Orion B9 star-forming region with the Effelsberg 100-m and APEX telescopes, respectively. These line observations were combined with our previous $870 \mu \mathrm{m}$ submm dust continuum data of the region. The data were used to determine the physical characteristics of dense cores in this region. We mainly investigated the temperatures and kinematics of the cores, and recalculated the temperature-dependent quantities presented in Paper I (Miettinen et al. 2009). Our main results and conclusions are as follows:

1. The gas kinetic temperatures of the cores are in the range 29.4-13.9 K, with an average value of about $12 \mathrm{~K}$. No significant difference in $T_{\text {kin }}$ was found between starless and protostellar cores. The temperature values are similar to those in nearby low-mass star-forming regions.

2. The cores are characterised by subsonic, or at most transonic, non-thermal motions. Thus, the cores are kinematically similar to those in nearby low-mass star-forming regions.

3 . In the case of protostellar cores, we found a positive correlation between the non-thermal $\mathrm{NH}_{3}$ linewidth and bolometric luminosity. This can be understood so that on one hand, the embedded central protostar enhances the level of turbulent motions within the parent core, and on the other hand, more turbulent initial conditions lead to the formation of more massive stars with higher luminosity.

4. The core masses are, on average, about $11 \%$ higher than those reported in Paper I when calculated by assuming that $T_{\text {dust }}=T_{\text {kin }}$ (in Paper I, we assumed that $T_{\text {dust }}=10 \mathrm{~K}$ for starless cores). These masses are very likely drawn from the 
same parent distribution as the core masses in the northern part of Orion B (Nutter \& Ward-Thompson 2007).

5. Almost all cores were found to be close to virial equilibrium when the internal kinetic pressure and self-gravity are taken into account. In addition, they are likely to be exposed to a substantial turbulent ram pressure from the intercore gas (as can be evaluated from previous ${ }^{13} \mathrm{CO}$ data), which is sufficient to bring them to equilibrium or make them collapse. In particular, it seems probable that most if not all starless cores detected in this survey are prestellar.

6. The fractional $\mathrm{NH}_{3}$ and $\mathrm{N}_{2} \mathrm{H}^{+}$abundances in the cores are $\sim 1.5-9.8 \times 10^{-8}$ and $\sim 0.2-5.9 \times 10^{-10}$, respectively. The $\mathrm{NH}_{3} / \mathrm{N}_{2} \mathrm{H}^{+}$column density ratio is higher in prestellar cores than protostellar cores. Similar trend has also been observed in other star-forming regions. The $\mathrm{NH}_{3}$ abundance appears to decrease with increasing gas density (as traced by $870 \mu \mathrm{m}$ continuum emission). This tendency is likely to be caused by the accretion of $\mathrm{NH}_{3}$ onto grains at very high densities, and conforms to the recent results of Friesen et al. (2009) in Ophiuchus.

7. A few cores have a much lower radial velocity compared to the systemic velocity of the region $\left(\sim 9 \mathrm{~km} \mathrm{~s}^{-1}\right)$, and do not necessarily belong to the same volume of the cloud as the rest of the cores. Instead, they are likely to be members of the "low-velocity" part of Orion B. This increases the relative number of pre- and protostellar cores in Orion B9 and consequently, the statistical lifetime of the prestellar phase of core evolution (by $25 \%$ in both cases).

8. Many of the properties of dense cores in Orion B9 suggest that their evolution is likely to be dynamic, i.e., comparable to the free-fall timescale. On the other hand, the new data and an improved analysis of spatial distribution have made the role of turbulent fragmentation in the core formation less evident than it appeared in Paper I. According to the new results, the cores are unlikely to be randomly positioned within the region, and they are not clustered in the way expected if the fragmentation is driven on large scale turbulence. Futhermore, the core properties are similar to those found in the regions of isolated star formation.

9. The Orion B9 region is likely to be influenced by the massive stars of the nearby Ori OB $1 \mathrm{~b}$ group. This explains the origin of the low-velocity material in the southern parts of Orion B (Wilson et al. 2005). Moreover, this interaction process could have led to formation of dense filaments, from which the dense cores were fragmented out by the action of gravitational instability.

The present paper demonstrates the importance of a kinematic and temperature information for the studies of dense cores and star formation. A lack of radial velocity information, for instance, may cause errors when examining the relative numbers of starless and protostellar cores. This, in turn, affects the lifetime estimates based on statistical arguments. The core lifetime is an important discriminator between different theories of core and star formation, particularly between the turbulence and ambipolar diffusion driven star formation scenarios.

Acknowledgements. We thank the referee for helpful comments, and the staff at the Effelsberg for their hospitality and support during our observations. We also thank the staff at the APEX telescope for performing the observations in the service mode. The authors acknowledge support from the Academy of Finland through grants 117206 and 132291 .

\section{References}

Aikawa, Y., Herbst, E., Roberts, H., \& Caselli, P. 2005, ApJ, 620, 330

Aikawa, Y., Wakelam, V., Garrod, R. T., \& Herbst, E. 2008, ApJ, 674, 984

Aoyama, H., Mizuno, N., Yamamoto, H., et al. 2001, PASJ, 53, 1053

Ballesteros-Paredes, J., Gazol, A., Kim, J., et al. 2006, ApJ, 637, 384

Bally, J. 2008, Handbook of Star Forming Regions, I, 459

Bally, J., Reipurth, B., \& Aspin, C. 2002, ApJ, 574, L79

Bertoldi, F., \& McKee, C. F. 1992, ApJ, 395, 140

Boulares, A., \& Cox, D. P. 1990, ApJ, 365, 544

Buckle, J. V., Curtis, E. I., Roberts, J. F., et al. 2010, MNRAS, 401, 204

Busquet, G., Palau, A., Estalella, R., et al. 2009, A\&A, 506, 1183

Caselli, P., \& Myers, P. C. 1995, ApJ, 446, 665

Caselli, P., Benson, P. J., Myers, P. C., \& Tafalla, M. 2002, ApJ, 572, 238

Caselli, P., Vastel, C., Ceccarelli, C., et al. 2008, A\&A, 492, 703

Evans, N. J., Dunham, M. M. Jørgensen, J. K., et al. 2009, ApJS, 181, 321

Flower, D. R., Pineau des Forêts, G., \& Walmsley, C. M. 2006, A\&A, 456, 215

Foster, J. B., Rosolowsky, E. W., Kauffmann, J., et al. 2009, ApJ, 696, 298

Friesen, R. K., di Francesco, J., Shirley, Y. L., \& Myers, P. C. 2009, ApJ, 697, 1457

Friesen, R. K., di Francesco, J., Shimajiri, Y., \& Takakuwa, S. 2010, ApJ, 708, 1002

Frieswijk, W. W. F., Spaans, M., Shipman, R. F., Teyssier, D., \& Hily-Blant, P. 2007, A\&A, 475, 263

Goldsmith, P. F., \& Langer, W. D. 1978, ApJ, 222, 881

Harju, J., Walmsley, C. M., \& Wouterloot, J. G. A. 1993, A\&AS, 98, 51

Harju, J., Haikala, L. K., Lehtinen, K., et al. 2006, A\&A, 454, L55

Ho, P. T. P., \& Townes, C. H. 1983, ARA\&A, 21, 239

Ho, P. T. P., Barrett, A. H., Myers, P. C., et al. 1979, ApJ, 234, 912

Hotzel, S., Harju, J., Lemke, D., Mattila, K., \& Walmsley, C. M. 2001, A\&A, 372,302

Ikeda, N., Kitamura, Y., \& Sunada, K. 2009, ApJ, 691, 1560

Jijina, J., Myers, P. C., \& Adams, F. C. 1999, ApJS, 125, 161

Johnstone, D., Fich, M., Mitchell, G. F., \& Moriarty-Schieven, G. 2001, ApJ, 559,307

Johnstone, D., Matthews, H., \& Mitchell, G. F. 2006, ApJ, 639, 259

Johnstone, D., Rosolowsky, E., Tafalla, M., \& Kirk, H. 2010, ApJ, 711, 655

Kauffmann, J., Bertoldi, F., Bourke, T. L., et al. 2008, A\&A, 487, 993

Kirk, H., Johnstone, D., \& Tafalla, M. 2007, ApJ, 668, 1042

Klein, B., Philipp, S. D., Krämer, I., et al. 2006, A\&A, 454, L29

Klessen, R. S. 2001, ApJ, 556, 837

Klessen, R. S., Heitsch, F., \& Mac Low, M.-M. 2000, ApJ, 535, 887

Kunz, M. W., \& Mouschovias, T. C. 2009, MNRAS, 399, L94

Lada, C. J., Muench, A. A., Rathborne, J., et al. 2008, ApJ, 672, 410

Larson, R. B. 1981, MNRAS, 194, 809

Lee, C.-F., Ho, P. T. P., Bourke, T. L., et al. 2008, ApJ, 685, 1026

Mac Low, M.-M., \& Klessen, R. S. 2004, Rev. Mod. Phys., 76, 125

Maruta, H., Nakamura, F., Nishi, R., Ikeda, N., \& Kitamura, Y. 2010, ApJ, 714 680

Miettinen, O., Harju, J., Haikala, L. K., et al. 2009, A\&A, 500, 845 (Paper I)

Myers, P. C., \& Benson, P. J. 1983, ApJ, 266, 309

Myers, P. C., Ladd, E. F., \& Fuller, G. A. 1991, ApJ, 372, L95

Nutter, D., \& Ward-Thompson, D. 2007, MNRAS, 374, 1413

Ott, M., Witzel, A., Quirrenbach, A., et al. 1994, A\&A, 284, 331

Padoan, P., \& Nordlund, A. 2002, ApJ, 576, 870

Pagani, L., Daniel, F., \& Dubernet, M.-L. 2009, A\&A, 494, 719

Peng, B., Kraus, A., Krichbaum, T. P., \& Witzel, A. 2000, A\&AS, 145, 1

Pillai, T., Wyrowski, F., Carey, S. J., \& Menten, K. M. 2006, A\&A, 450, 569

Rosolowsky, E. W., Pineda, J. E., Foster, J. B., et al. 2008, ApJS, 175, 509

Schnee, S., Rosolowsky, E., Foster, J., et al. 2009, ApJ, 691, 1754

Shetty, R., Kauffmann, J., Schnee, S., Goodman, A. A., \& Ercolano, B. 2009, ApJ, 696, 2234

Tafalla, M., Myers, P. C., Caselli, P., Walmsley, C. M., \& Comito, C. 2002, ApJ, 569,815

Tafalla, M., Myers, P. C., Caselli, P., \& Walmsley, C. M. 2004, A\&A, 416, 191

Tafalla, M., Santiago-García, J., Myers, P. C., et al. 2006, A\&A, 455, 577

Vassilev, V., Meledin, D., Lapkin, I., et al. 2008, A\&A, 490, 1157

Vázquez-Semadeni, E., Kim, J., Shadmehri, M., \& Ballesteros-Paredes, J. 2005, ApJ, 618, 344

Wilson, B. A., Dame, T. M., Masheder, M. R. W., \& Thaddeus, P. 2005, A\&A, 430,523

Wiseman, J., Wootten, A., Zinnecker, H., \& McCaughrean, M. 2001, ApJ, 550, L87 